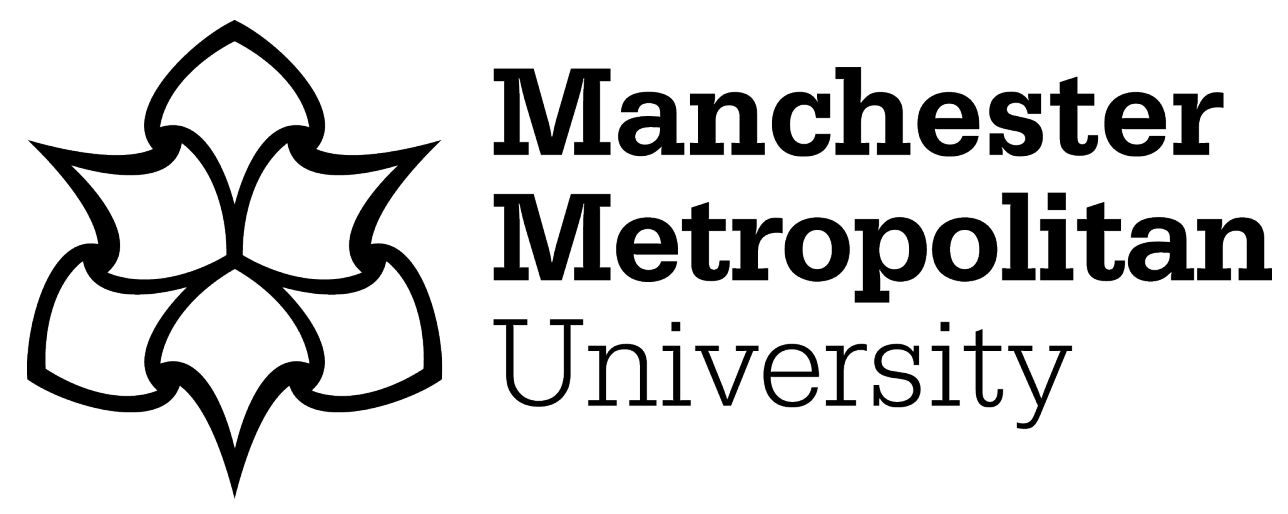

Miller, R, Griffiths, PT, Hussain, Z ORCID logoORCID: https://orcid.org/00000001-6756-6058 and Garrett, SJ (2020) On the stability of a heated rotatingdisk boundary layer in a temperature-dependent viscosity fluid. Physics of Fluids, 32 (2). 024105-024105. ISSN 1070-6631

Downloaded from: https://e-space.mmu.ac.uk/625075/

Version: Accepted Version

Publisher: AIP Publishing

DOI: https://doi.org/10.1063/1.5129220

Please cite the published version 


\title{
On the stability of a heated rotating-disk boundary layer in a temperature-dependent viscosity fluid
}

\author{
R. Miller ${ }^{1}$ \\ P. T. Griffiths ${ }^{2}$ \\ Z. Hussain ${ }^{3}$ \\ S. J. Garrett ${ }^{4}$ \\ ${ }^{1}$ School of Engineering, University of Leicester, Leicester, LE1 7RH, UK \\ ${ }^{2}$ Centre for Fluid and Complex Systems, Coventry University, Coventry, CV1 5FB, UK \\ ${ }^{3}$ Centre for Mathematical Modelling \& Flow Analysis, Manchester Metropolitan University, Manchester, M1 5GD, \\ UK \\ ${ }^{4}$ School of Mathematics \& Actuarial Science, University of Leicester, Leicester, LE1 7RH, UK
}

January 6, 2020

\begin{abstract}
The paper presents a linear stability analysis of the temperature-dependent boundary-layer flow over a rotating disk. Gas- and liquid-type responses of the viscosity to temperature are considered, and the disk rotates in both a quiescent and an incident axial flow. Temperature-dependent-viscosity flows are typically found to be less stable than the temperature independent cases, with temperature dependences that produce high wall viscosities yielding the least stable flows. Conversely, increasing the incident axial flow strength produces greater flow stability. Transitional Reynolds numbers for these flows are then approximated through an $e^{N}$-type analysis, and are found to vary in approximate concordance with the critical Reynolds number. Examination of the component energy contributions shows that flow stability is affected exclusively through changes to the mean flow. The results are discussed in the context of Chemical Vapour Deposition reactors.
\end{abstract}

\section{Introduction}

A convectively unstable flow is one featuring the manifestation of small disturbances in an otherwise laminar state. These convective instabilities develop and grow downstream, eventually gaining sufficient amplitude to trigger non-linear behaviour that causes the flow to transition from the laminar to turbulent regime. As such, there is a long history of interest in the mechanisms and control of these instabilities. The effects of small disturbances on the laminar flow over a flat plate were first investigated by Tollmien [33] and Schlichting [32], who considered the amplification of linear, wave-like disturbances (aptly named Tollmien-Schlichting waves) as the primary mechanism for flow instability. Later, Gregory et al. [11] investigated the formation of co-rotating stationary vortices (crossflow instabilities) on the rotating disk.

The physics of a rotating disk situated in a stationary fluid can be described as a centrifugal pump or fan. As the disk rotates, viscous effects cause the surrounding fluid to rotate with it. The fluid is dragged down towards the disk surface before flowing radially outwards to be cast off at the disk edge. The seminal work of von Kármán [22] found exact similarity solutions to the Navier-Stokes equations for the infiniteplane rotating disk problem, allowing them to be reduced to a set of ordinary differential equations that characterise the mean flow.

Much of the early motivation for the study of the stability of the rotating-disk flow is drawn from aerospace engineering; both the rotating disk and the swept wing exhibit inflectional mean-flow profiles susceptible to crossflow instabilities. These arise from inviscid effects and manifest as spiral vortices as first observed experimentally by Gray [10] for a swept wing and later Gregory et al. [11] for the rotating disk. The rotating disk, however, proved advantageous for the study of crossflow instabilities due to its axisymmetric geometry and exact similarity solutions of the Navier-Stokes equations allowing for significantly simplified mathematical investigation when compared to the swept wing. The study of rotating-disk flows has subsequently developed into its own field, with direct industrial applications as well as remaining a model flow of fundamental interest.

One of the key differences between the swept wing and the rotating disk boundary layers is that the latter is subject to Coriolis forces. Malik [29] discovered an additional instability mechanism arising from this by performing a numerical analysis to compute the neutral curves for stationary instability modes in the rotatingdisk boundary layer. This work was the first departure from the previous fourth-order Orr-Sommerfeld type analyses in favour of a sixth-order system that includes rotational Coriolis and viscous terms. The work, 
also verified asymptotically by Hall [12], found that two branches exist: the previously known upper branch associated with crossflow instability (type I), and a lower branch associated with streamline curvature and the balancing of Coriolis and viscous forces (type II). The type II mode has been found in all subsequent studies and is known to be particularly important when travelling modes are permitted, see for example Hussain et al. [16]. Observing the type II disturbance experimentally has however proved difficult as the type I mode is almost always dominant for all practical flows where unavoidable roughness acts to select modes that rotate with the disk surface. The work presented by Fedorov et al. [8] is often pointed to as a rare observation of the type II mode and more recent experimental work [26,6] has shown the potential for travelling modes to be selected.

This paper considers the linear convective stability behaviour of flows over the rotating disk under a temperature-dependent viscosity model and is closely related to the sister publication Miller et al. [31] concerning the Blasius/Falkner-Skan boundary layer stability under the same viscosity model. There are however a number of key physical differences between the stability of the von Kármán and Blasius/Falkner-Skan boundary layers. Notably, while rotation of the disk is sufficient for a potentially unstable boundary layer due to the inviscid nature of the crossflow instability, Tollmien-Schlichting waves are generated through viscous effects and as such external forcing is required to generate a boundary layer (and therefore instability) over the flat plate. The two-dimensional nature of the plate problem also renders all instabilities 'travelling', with no direct analogue to the stationary vortices formed on the disk. Nevertheless, the methodology presented here is much the same as that presented in Miller et al. [31] and the interested reader is referred there for an in-depth discussion regarding two-dimensional temperature dependent boundary layer flows.

Whilst normal-mode linear stability analyses typically aim to model the early stages of transitional flows, numerous studies have highlighted the importance of nonlinearity and non-normality in the prediction of transition to turbulence, as well as their role in the formation of primary instabilities. The $e^{N}$ analysis developed by van Ingen [17] (reviewed extensively in van Ingen [18] and utilised in this paper) is often used to consolidate nonlinearity within the linear framework, utilising the linear amplification rates to find empirical agreement with transitional Reynolds numbers observed in experiments. Bertolotti et al. [2] utilise parabolic stability equations to examine both non-parallel and nonlinear effects on the Blasius boundary layer stability, concluding that whilst both are destabilising with reference to the linear results, neither is significant enough to account for the discrepancies between neutral curve data obtained from theory and experiments.

Non-normal studies have allowed for the investigation of global instabilities in boundary layers and their role in transition. In response to the assertion of Huerre \& Monkewitz [15] that a linear analysis is sufficient for investigating global behaviour, Chomaz [4] identifies that instability behaviour becomes rapidly nonlinear beyond a global threshold. Lingwood [25] provided the benchmark for the absolute instability of the rotating disk boundary layer flow, finding theoretical and experimental agreement of the flow becoming absolutely unstable at a Reynolds number of 510 and transition to turbulence at 513. From this it was concluded that absolute instability acts as a linear threshold, beyond which non-linear behaviour in the flow begins to dominate and develops into fully turbulent flow. Briggs' pinching criterion states that the absolutely unstable parameters must lie within the range of those that are convectively unstable (that is, the absolute neutral stability curve is contained within the convective neutral curve). To this end, Healey [13] shows that the absolute neutral stability curve associated with the rotating disk flow also exhibits the two branch structure of its convective equivalent. Utilising front propagation theory and direct numerical simulation (DNS), Cossu et al. [7] examine the nonlinear behaviour of disturbances beyond the threshold for global instability, finding that for large-amplitude impulses the globally unstable behaviour of the Blasius boundary layer is decisively nonlinear, yet the wave front speed is identical to that of its linear counterpart. The findings of Healey [13] and Cossu et al. [7] lead to the conclusion that conducting local linear analyses of unstable flows over both the disk and the plate is still of importance when extending to a global stability analysis to inform the nonlinear behaviour. Whilst nonlinearity, non-normality and absolute instability are beyond the scope of the work presented here, it is acknowledged that they form an active area of research that is greatly informed by the behaviour of convectively unstable flows. As such, the work presented here and in Miller et al. [31] can be considered as a first step towards understanding the global criteria for instability and transition in modified von Kármán and Blasius/Falkner-Skan flows. The interested reader is directed to Chomaz [4] for an extensive review of non-normal, nonlinear and global studies and their role in boundary layer stability. In addition, the review presented by Lingwood \& Alfredsson [27] includes recent developments regarding absolute instability over rotating disk boundary layers.

The significance of viscous effects on flow instability has led to interest in variable viscosity flows. Strong temperature gradients can cause the viscosity of a fluid to vary immensely and, as such, there is a great deal of literature studying temperature dependent viscosity flows. Ling \& Dybbs [24] assert that an inverselinear relationship between temperature and viscosity accurately models the behaviour of a range of real fluids. Kafoussias \& Williams [21] utilise this relationship in their study of the free-forced vertical flow with 
temperature dependent viscosity over a flat plate. There, it is found that fluids that become more viscous with increasing temperature create both a narrower temperature profile and velocity profile, whilst the effect is reversed for fluid viscosities that decrease with temperature. Wall \& Wilson [34] consider the effects of two different exponential-type viscosity temperature relationships on Blasius flow stability. In both models they note that for an isothermally heated plate, an exponentially-decaying viscosity produces a destabilised flow, whilst an exponentially-increasing viscosity is more stable. An inverse linear temperature dependent viscosity law is also considered by Jasmine \& Gajjar [19] in their study of flow stability over a rotating disk and similar qualitative effects as cited by Kafoussias \& Williams [21] are observed, as well as finding that a viscosity that decreases more rapidly with increasing temperature yields a less stable flow. Again, this broadly agrees with the findings of Wall \& Wilson [34]. The work presented in this paper considers the inverse-linear relationship between viscosity and temperature utilised by Ling \& Dybbs [24], Kafoussias \& Williams [21] and Jasmine \& Gajjar [19], amongst others.

There is a wealth of literature concerning flows over rotating media, most of which set the fluid as otherwise stationary. More recent studies examine the effects of an oncoming flow over rotating media to better understand transitional flows in industrially relevant configurations. Chen \& Mortazavi [3] produce a model of a Chemical Vapour Deposition (CVD) reactor featuring a forced flow parameter to represent the injection of gas into the system. Garrett et al. [9] present a linear stability analysis (both asymptotic and numerical) of the enforced axial flow over a rotating cone, where it is found that a stronger axial flow results in delaying the onset of transition in both modes of instability. This is extended to the flow over a rotating disk in Hussain et al. [16], where a similar result is found.

The work presented in this paper has particular relevance to vertical CVD systems where it is important that laminar flow is maintained over a heated substrate rotating in a forced flow. This is necessary to promote regular and cohesive film growth. Longitudinal roll instabilities are well established in buoyancy-driven flows with a free convection element [28] and have been observed in CVD reactors at Rayleigh numbers* beyond 1708 [14]. Whilst there is a great deal of literature regarding solutions to the laminar flow in a CVD reactor, there is (to the authors' knowledge) no such study on the existence of unstable or transitional flows due to forced convection. Convective instabilities arising from forced flow are perhaps not considered due to a dichotomic view of the flow being laminar or turbulent. Typically the operating Reynolds number of a vertical CVD reactor is in the range of 1 to $200[20,14]$, which most would consider to be firmly in the laminar regime. However, modifications to the stability solutions of flows over both the disk and the plate have shown that destabilising effects are possible, as demonstrated by Wall \& Wilson [34] where a critical Reynolds number of approximately 220 is achieved at one extreme of their viscosity temperature dependence parameter. Hence, it is possible that certain destabilising criteria may be sufficiently influential to produce primary instabilities within the operational parameters of a CVD reactor. It is widely accepted that the steep temperature gradients in a CVD reactor cause variation in all physical properties of the fluid [23, 20, 14], supporting the need for a temperature dependent viscosity model, and as such this study serves as the first step towards a larger stability analysis of flows in CVD reactors.

In particular, the work presented here considers the convective instability of the boundary layer resulting from the forced flows of fluids with temperature dependent viscosity incident on a heated rotating disk. It proceeds as follows: In $\S 2$ we formulate and solve the equations representing the steady, mean flow of the fluid over the disk. The linear convective stability of these flows is then assessed in $\S 3$, via the formulation of neutral stability curves and $e^{N} \&$ integral energy analyses. Finally, our conclusions are discussed in $§ 4$.

\section{Problem formulation and mean-flow analysis}

\subsection{Derivation of the mean-flow equations}

Consider a heated disk of infinite radius, rotating about its centre of axis $z^{*}$ with angular velocity $\boldsymbol{\Omega}^{*}$. The disk is situated perpendicularly downstream from an incompressible Newtonian fluid. As the disk rotates, fluid is entrained towards its surface and moves with velocity $\mathbf{U}^{*}=\left(u^{*}, v^{*}, w^{*}\right)$, representing boundary-layer velocities in the radial, azimuthal and axial directions $\left(r^{*}, \theta\right.$ and $\left.z^{*}\right)$ respectively. The disk is heated to a temperature $T_{w}^{*}$ whilst the temperature of the fluid in the free stream is $T_{\infty}^{*}$; the fluid in the boundary layer has temperature $T^{*}$. Note that in all that follows an asterisk indicates a dimensional quantity.

In the rotating frame of reference, the flow can be described by the Navier-Stokes equations in cylindrical

${ }^{*}$ The Rayleigh number is the product of the Grashof and Prandtl numbers, i.e. the ratio of buoyancy and viscous forces multiplied by the ratio of momentum and thermal diffusivities. 
polar coordinates,

$$
\begin{aligned}
\left(\frac{\partial}{\partial t^{*}}+\mathbf{U}^{*} \cdot \nabla^{*}\right) \mathbf{U}^{*}+\underbrace{2 \boldsymbol{\Omega}^{*} \times \mathbf{U}^{*}}_{R_{1}}+\underbrace{\boldsymbol{\Omega}^{*} \times \mathbf{U}^{*} \times \mathbf{r}^{*}}_{R_{2}} & =0, \frac{1}{\rho^{*}} \nabla^{*} p^{*}+\frac{1}{\rho^{*}} \nabla^{*} \cdot \boldsymbol{\tau}^{*}, \\
\left(\frac{\partial}{\partial t^{*}}+\mathbf{U}^{*} \cdot \nabla^{*}\right) T^{*} & =\frac{k^{*}}{\rho^{*} C_{p}^{*}} \nabla^{* 2} T^{*} .
\end{aligned}
$$

where $t^{*}$ is time, $\boldsymbol{\Omega}^{*}=\left(0,0, \Omega^{*}\right)$ is the angular velocity vector, $\mathbf{r}^{*}=(r, 0,0)$ is the radial position vector, $\rho^{*}$ is the density, $p^{*}$ is pressure, $k^{*}$ is the thermal diffusion coefficient and $C_{p}^{*}$ is the specific heat of the fluid. The viscous stress tensor is $\boldsymbol{\tau}^{*}=\mu^{*} \dot{\gamma}^{*}$, where $\mu^{*}$ is the viscosity and $\dot{\boldsymbol{\gamma}}^{*}=\left[\nabla^{*} \mathbf{U}^{*}+\nabla^{*}\left(\mathbf{U}^{*}\right)^{\mathrm{T}}\right]$ is the rate-of-strain tensor. The terms labelled $R_{1}$ and $R_{2}$ are rotational terms arising from Coriolis and centrifugal acceleration forces, respectively. The heat continuity equation (1c) is coupled to equations (1a) and (1b) via the imposed temperature dependence of the fluid viscosity

$$
\mu^{*}=\frac{\mu_{\infty}^{*}}{1+\varepsilon^{*}\left(T^{*}-T_{\infty}^{*}\right)} .
$$

Here $\mu_{\infty}^{*}$ is the fluid viscosity in the free stream and $\varepsilon^{*}$ is the temperature dependence constant in inverse temperature units. Note that $\varepsilon^{*}$ is an intrinsic property of the fluid and changing $\varepsilon^{*}$ would represent a fundamentally different fluid. For an isothermally heated disk (as considered here), $\varepsilon^{*}>0$ represents the viscothermal behaviour of a liquid (a fluid that becomes less viscous with increasing temperature), whilst $\varepsilon^{*}<0$ represents that of a gas (a fluid that becomes more viscous with increasing temperature, with appropriate consideration of parameters to ensure positive viscosity). Applying $\varepsilon=0$ returns a constant viscosity and uncouples equation (1c) from equations (1a) and (1b). Ling \& Dybbs [24] cite this expression for $\mu^{*}$ as an accurate representation of the relationship between viscosity and temperature for a range of real fluids such as water and crude oil; this motivates its use here.

The motion of the fluid is influenced both by the rotation of the disk and the axially enforced element towards the disk surface. The enforced axial flow component is derived from the radial pressure balance at the boundary layer edge

$$
U_{e}^{*} \frac{\partial U_{e}^{*}}{\partial r^{*}}=-\frac{1}{\rho^{*}} \frac{\partial p^{*}}{\partial r^{*}}
$$

where $U_{e}^{*}=C^{*} r^{*}$ is the flow velocity at the boundary-layer edge. The constant $C^{*}$ represents the strength of the enforced flow in inverse time units.

We non-dimensionalise (1) by the scaling variables

$$
\begin{aligned}
& \mathbf{U}^{*}=(u, v, \delta w) U_{\infty}^{*}, \quad\left(r^{*}, z^{*}\right)=(r, \delta z) L^{*}, \quad t^{*}=\frac{t L^{*}}{U_{\infty}^{*}}, \\
& p^{*}=p\left(U_{\infty}^{*}\right)^{2} \rho^{*}, \quad T^{*}-T_{\infty}^{*}=T\left(T_{w}^{*}-T_{\infty}^{*}\right), \quad \varepsilon^{*}=\frac{\varepsilon}{T_{w}^{*}-T_{\infty}^{*}},
\end{aligned}
$$

where $U_{\infty}^{*}=L^{*} \Omega^{*}$, with $L^{*}$ defined as a generic length scale. The boundary-layer scaling constant is $\delta$. We note that setting $\varepsilon$ as greater or less than 0 remains representative of liquid- or gas-type viscosity behaviour, respectively, consistent with the definition of $\varepsilon^{*}$.

The laminar flow is steady and axisymmetric, meaning that derivatives with respect to $t$ and $\theta$ are neglected. We define the Reynolds number as $R e=U_{\infty}^{*} L^{*} \rho^{*} / \mu_{\infty}^{*}$ and, with $R e \gg 1$, determine the governing equations at leading order to be

$$
\begin{aligned}
\frac{1}{r} \frac{\partial(r u)}{\partial r}+\frac{\partial w}{\partial z} & =0 \\
u \frac{\partial u}{\partial r}+w \frac{\partial u}{\partial z}-\frac{(v+r)^{2}}{r} & =r T_{s}^{2}+\frac{1}{\delta^{2} R e}\left(\mu \frac{\partial^{2} u}{\partial z^{2}}+\frac{\partial \mu}{\partial z} \frac{\partial u}{\partial z}\right), \\
u \frac{\partial v}{\partial r}+w \frac{\partial v}{\partial z}+\frac{u v}{r}+2 u & =\frac{1}{\delta^{2} R e}\left(\mu \frac{\partial^{2} v}{\partial z^{2}}+\frac{\partial \mu}{\partial z} \frac{\partial v}{\partial z}\right), \\
u \frac{\partial w}{\partial r}+w \frac{\partial w}{\partial z} & =-\frac{1}{\delta^{2}} \frac{\partial p}{\partial z}+\frac{1}{\delta^{2} R e}\left(\mu \frac{\partial^{2} w}{\partial z^{2}}+2 \frac{\partial \mu}{\partial z} \frac{\partial w}{\partial z}\right), \\
u \frac{\partial T}{\partial r}+w \frac{\partial T}{\partial z} & =\frac{1}{\delta^{2} \operatorname{Pr} R e} \frac{\partial^{2} T}{\partial z^{2}},
\end{aligned}
$$


where it is concluded that $\delta=\mathcal{O}\left(R e^{-1 / 2}\right)$ and the boundary-layer thickness is $\delta^{*}=\delta L^{*}=\sqrt{\mu_{\infty}^{*} / \rho^{*} \Omega^{*}}$. Note that $\operatorname{Pr}=C_{p}^{*} \rho^{*} / k^{*}$ is the Prandtl number that is henceforth set to $\operatorname{Pr}=0.72$. This is a suitable value for various fluids including air.

Imposing the similarity solutions

$$
u=r U(z), \quad v=r V(z), \quad w=W(z), \quad p=P(z), \quad T=\Theta(z),
$$

leads to a modified form of the von Kármán equations

$$
\begin{aligned}
2 U+W^{\prime} & =0, \\
U^{2}+U^{\prime} W-(V+1)^{2}-\left(\bar{\mu} U^{\prime}\right)^{\prime}-T_{s}^{2} & =0, \\
2 U(V+1)+V^{\prime} W-\left(\bar{\mu} V^{\prime}\right)^{\prime} & =0, \\
W W^{\prime}+P^{\prime}-\bar{\mu} W^{\prime \prime}-2 \bar{\mu}^{\prime} W^{\prime} & =0, \\
\operatorname{Pr} W \Theta^{\prime}-\Theta^{\prime \prime} & =0,
\end{aligned}
$$

where $\bar{\mu}=1 /(1+\varepsilon \Theta)$ and primes indicate differentiation with respect to $z$. We note that (5d) is obtained from a leading order expansion of $P(z)$ in terms of $\delta$. At leading order one finds that $P(z)=P(z=0)=P_{0}$. The solution at the next order is then determined from $(5 \mathrm{~d})$. The system is subject to the boundary conditions

$$
\begin{array}{r}
U(0)=V(0)=W(0)=P(0)=\Theta(0)-1=0, \\
U(z \rightarrow \infty) \rightarrow T_{s}, \quad V(z \rightarrow \infty) \rightarrow-1, \quad \Theta(z \rightarrow \infty) \rightarrow 0
\end{array}
$$

which reflect the no-slip criteria and Coriolis force balance conditions in the rotating frame. Note that the condition for the outer radial velocity is modified according to the pressure balance condition at the boundary-layer edge due to the enforced axial flow. The conditions for $\Theta(0)$ and $\Theta(z \rightarrow \infty)$ are indicative of a heated disk.

The familiar temperature-independent problem is returned when $\varepsilon=0$, and enforced axial flow is eliminated when $T_{s}=0$. Setting $\varepsilon=T_{s}=0$ returns the standard von Kármán equations.

With the exception of $(5 \mathrm{~d})$, the system $(5)$ is solved utilising a Newton-Raphson searching routine to find a suitable condition for the unknowns $U^{\prime}(0)$ and $V^{\prime}(0)$, alongside a double precision, fourth-order RungeKutta integration scheme to solve the mean flow functions through the boundary layer. Convergence is reached at $z_{\max }=20$ to a tolerance of $10^{-7}$, which can be considered representative of the free stream, though plots will be truncated to $z=10$ to better visualise the effects of varying the free parameters. Note that $(5 \mathrm{~d})$ is independently solvable for the temperature independent case, i.e. $\varepsilon=0$,

$$
P-P_{0}=-\left(2 U+\frac{W^{2}}{2}\right)
$$

with the stipulation that $P(z=0)=P_{0}$. However, the introduction of a temperature dependent viscosity leads to an additional term owing to the viscous stress tensor, $2 \bar{\mu}^{\prime} W^{\prime}$, and an incomplete solution that requires additional numerical integration is obtained

$$
P=-\left(2 \bar{\mu} U+\frac{W^{2}}{2}\right)+\int \bar{\mu}^{\prime} W^{\prime} \mathrm{d} z,
$$

in this case the $P_{0}$ term is absent because, as noted above, it is obtained in the leading order expansion of $P(z)$.

\subsection{Mean-flow profiles}

\subsubsection{Temperature-dependent viscosity (no axial flow)}

Figure 1 shows the resulting mean-flow profiles for a range of $\varepsilon$ values when $T_{s}=0$. We see that $\bar{\mu}$ is uniformally 1 for the temperature independent case of $\varepsilon=0$. For $\varepsilon \neq 0$, the viscosity converges on $\bar{\mu}(z \rightarrow$ $\infty)=1$; this is due to the uniform temperature in the free stream. Prior to this convergence, the viscosity is everywhere greater or less than one, i.e. the viscosity is increased or decreased throughout the boundary layer, for $\varepsilon<0$ and $\varepsilon>0$, respectively.

Increasing the value of $\varepsilon$ is found to move the radial velocity profile, $U(z)$, closer to the disk surface. This can be interpreted as a narrowing of the fluid boundary layer. Increasing $\varepsilon$ also reduces the viscosity at the 

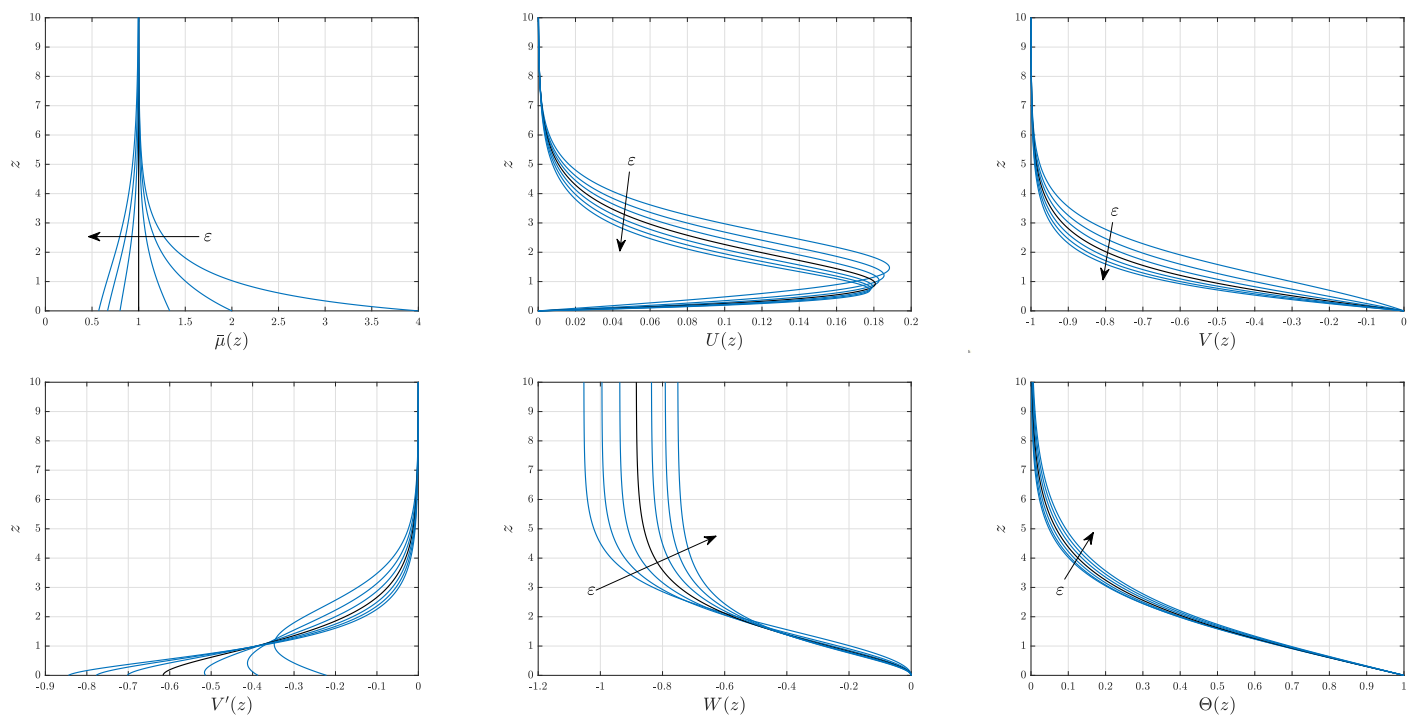

Figure 1: Mean-flow profiles for $-0.75 \leq \varepsilon \leq 0.75$ in 0.25 increments. The black lines indicate the temperature independent $(\varepsilon=0)$ solution.

wall, reducing the wall shear stress and the viscous interaction between the disk and the fluid. Hence, the ability of the disk to accelerate the fluid is diminished, resulting in a reduced $U(z)$ profile jet.

The azimuthal profile, $V(z)$, reaches convergence at a reduced value of $z$ as $\varepsilon$ is increased and is further evidence of the narrowing effect. This is consistent with the absolute value of $V^{\prime}(z)$ at the wall increasing with $\varepsilon$, showing that $V(z)$ grows more significantly with increasing distance from the disk surface. An interesting detail of the $V^{\prime}(z)$ profile is that it becomes inflectional for $\varepsilon<0$, meaning that there is a small region for which the azimuthal velocity increases linearly through the boundary layer, sharply increasing close to the disk surface and more steadily at a greater distance. This is of particular relevance to the results presented in $§ 3.5$ and are discussed in further detail there.

Figure 1 also demonstrates that increases in $\varepsilon$ reduces the vertical acceleration of $W(z)$ in the region close to the disk, as well as the converging outflow velocity closer to the free stream. The continuity equation suggests that this axial response is related to the effects of $\varepsilon$ on the radial profile. We interpret this physically as a result of the reduced viscosity inhibiting the centrifugal pump effect.

In contrast to the behaviour of the velocity profiles, the mean temperature profile, $\Theta(z)$, moves away from the disk surface with $\varepsilon$. Mathematically, this is a consequence of the $W(z)$-dependent term in (5e): as $\varepsilon$ is increased, the term is decreased due to the reduction in $W(z)$. In turn, $\Theta^{\prime}(z)$ is reduced, resulting in a shallower gradient in the $\Theta(z)$ profile. As such, whilst the momentum boundary layer is narrowed, increasing $\varepsilon$ results in a broadening of the thermal boundary layer, meaning the reduction in outflow must be favourable to the transferral of heat from the disk to the fluid.

Where values of $\varepsilon$ match, the solutions presented here are in exact agreement with those of Jasmine \& Gajjar [19].

\subsubsection{Enforced axial flow (no temperature dependence)}

Figure 2 shows mean-flow solutions for a range of $T_{s}$ values for $\varepsilon=0$. The prominent feature of the radial velocity profile, $U(z)$, is the free stream convergence to the applied value of $T_{s}$ according to the boundary condition outlined in (6). The maximum value of $U(z)$ increases with increasing axial flow strength, although the characteristic inflection is suppressed for higher axial flow strengths. This maximum also occurs at a moderately increased distance from the disk surface as the axial flow strength is increased. The profiles then converge to the boundary condition at a reduced distance, which is interpreted as a gentle narrowing of the boundary layer (consistent with the observed effects of temperature dependence).

The azimuthal velocity profile, $V(z)$, moves closer to the disk surface with increasing axial flow strength. Recall that the axial flow parameter $T_{s}$ represents the ratio of forced flow to the rotational speed of the disk. As such, increasing $T_{s}$ inhibits the influence of the disk's rotation on the flow, meaning that the fluid converges to zero rotational velocity $(V(z) \rightarrow 0)$ at a reduced distance from the disk surface.

The axial flow profiles, $W(z)$, exhibit a change in shape for $T_{s} \neq 0$ and become unbounded with increasing distance from the disk. Whilst acceleration in the axial direction is to be expected with enforced axial flow, the outflow velocity should still, in reality, converge at a certain distance from the disk surface. This unphysical unbounded acceleration is instead a result of the boundary condition imposed at $U(z \rightarrow \infty)$. However, the 

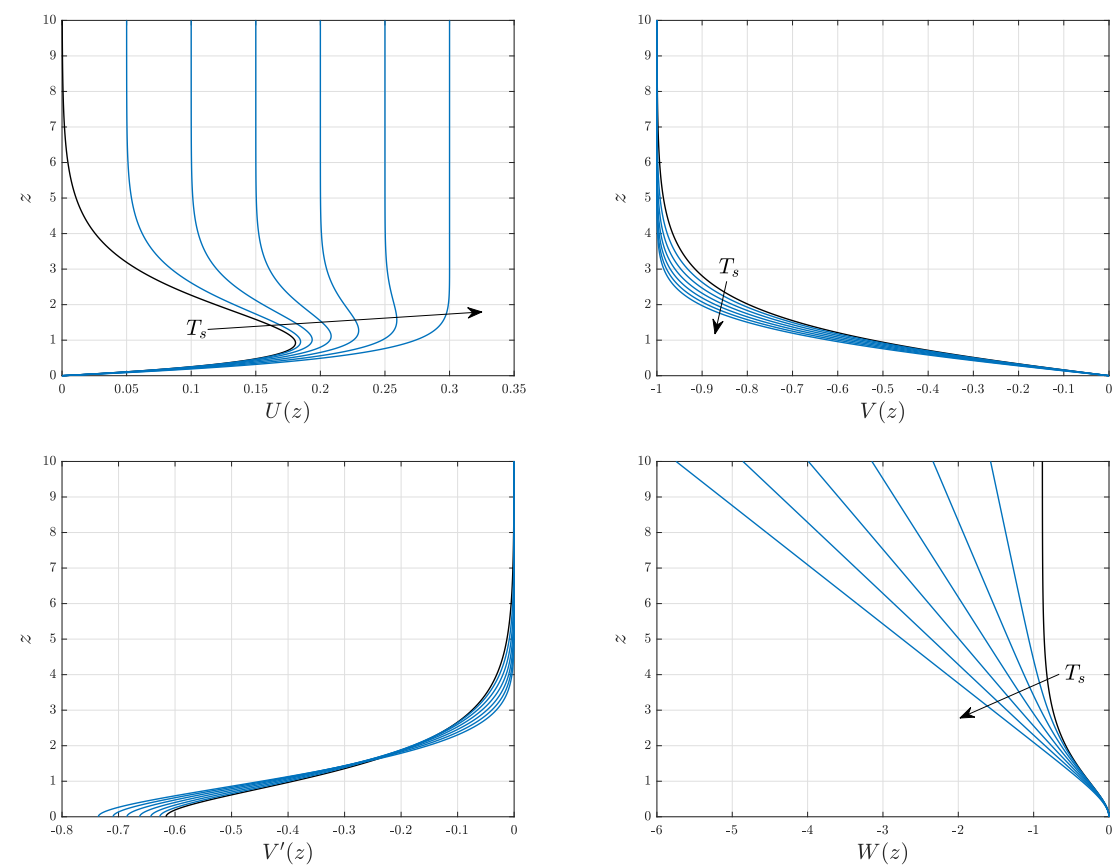

Figure 2: Mean-flow profiles for the range $0 \leq T_{s} \leq 0.3$ in 0.05 increments. The black lines indicate the zero axial enforcement $\left(T_{s}=0\right)$ solution.

physical element of increased outflow does support the narrowing of the boundary layer, as fluid is ejected from the disk surface more rapidly. This is discussed further by Hussain et al. [16] and, where parameter values match, the solutions presented here are in exact agreement with those presented there.

\subsubsection{Combined effects}
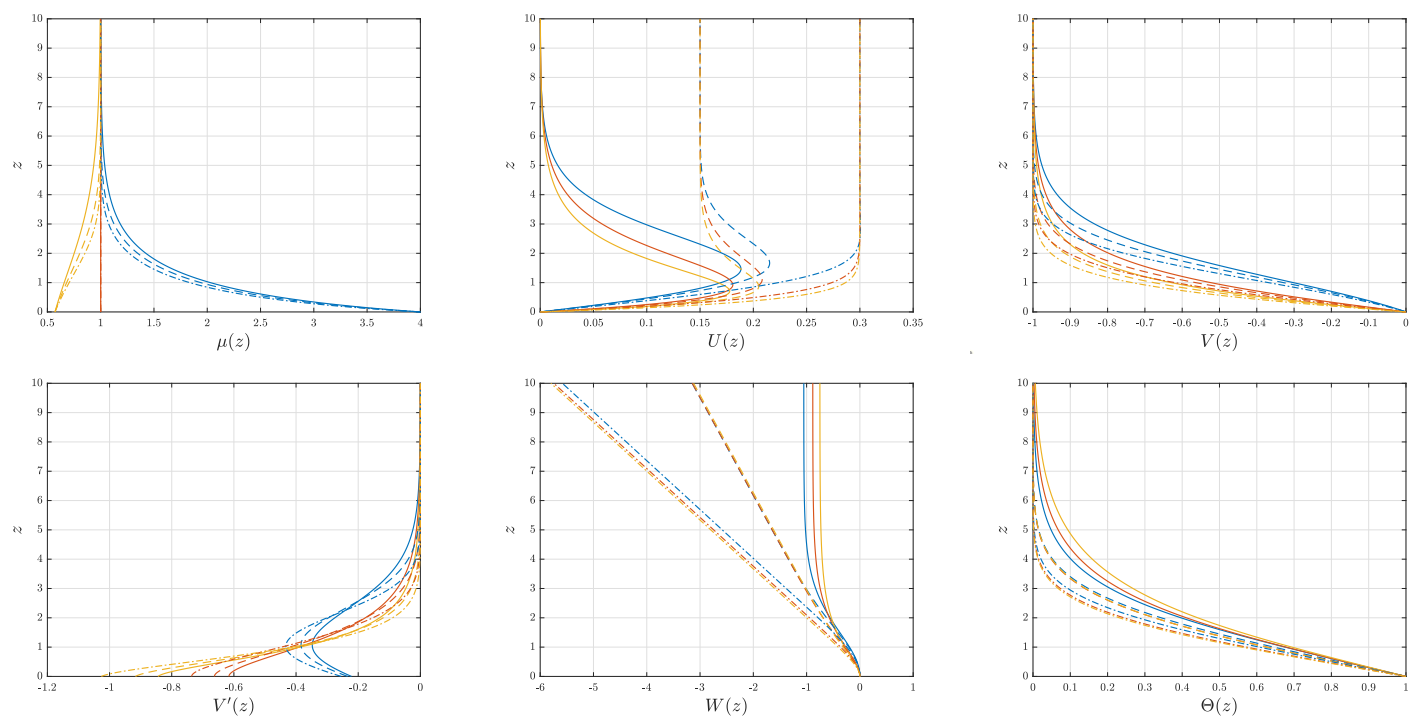

Figure 3: Mean-flow profiles for a range of temperature dependencies and axial flow strengths. Solid lines $(-) T_{s}=0$; dashed lines $(--) T_{s}=0.15$; dash-dot lines $(-\cdot) T_{s}=0.3$. Line colours represent $\varepsilon=-0.75$ (blue), 0 (red) and 0.75 (yellow) respectively.

Figure 3 shows the combined effects of a temperature dependent viscosity and enforced axial flow on the mean flow over the rotating disk.

The interaction of the two effects is most clearly observed in the viscosity profiles. As the axial flow strength is increased, the viscosity profiles for the cases when $\varepsilon \neq 0$, move closer to the disk surface. The change in viscous behaviour then becomes localised to the region very close to the disk surface and the viscosity converges to that of the free stream over a reduced distance. This is consistent with the narrowing 


\begin{tabular}{|c|c|c|c|c|c|}
\hline \multicolumn{6}{|c|}{$T_{s}=0$} \\
\hline$\varepsilon$ & $\overline{U_{\max }}$ & $\overline{U^{\prime}(0)}$ & $\overline{V^{\prime}(0)}$ & $\overline{\Theta^{\prime}(0)}$ & $W(z \rightarrow \infty)$ \\
\hline-0.75 & 0.1884 & 0.2282 & -0.2216 & -0.3279 & -1.0531 \\
\hline-0.50 & 0.1855 & 0.3520 & -0.3869 & -0.3334 & -0.9951 \\
\hline-0.25 & 0.1828 & 0.4397 & -0.5136 & -0.3322 & -0.9377 \\
\hline 0.00 & 0.1808 & 0.5102 & -0.6159 & -0.3286 & -0.8845 \\
\hline 0.25 & 0.1794 & 0.5710 & -0.7022 & -0.3239 & -0.8358 \\
\hline 0.50 & 0.1784 & 0.6254 & -0.7773 & -0.3188 & -0.7915 \\
\hline 0.75 & 0.1779 & 0.6753 & -0.8444 & -0.3135 & -0.7511 \\
\hline \multicolumn{6}{|c|}{$T_{s}=0.15$} \\
\hline$\varepsilon$ & $\overline{\overline{U_{\max }}}$ & $\overline{U^{\prime}(0)}$ & $\overline{V^{\prime}(0)}$ & $\overline{\Theta^{\prime}(0)}$ & $\bar{~} \overline{W(z \rightarrow \infty)}$ \\
\hline-0.75 & 0.2153 & 0.2365 & -0.2340 & -0.3588 & $\sim$ \\
\hline-0.50 & 0.2127 & 0.3684 & -0.4117 & -0.3717 & $\sim$ \\
\hline-0.25 & 0.2103 & 0.4625 & -0.5499 & -0.3775 & $\sim$ \\
\hline 0.00 & 0.2083 & 0.5381 & -0.6626 & -0.3806 & $\sim$ \\
\hline 0.25 & 0.2069 & 0.6029 & -0.7583 & -0.3824 & $\sim$ \\
\hline 0.50 & 0.2058 & 0.6607 & -0.8419 & -0.3836 & $\sim$ \\
\hline 0.75 & 0.2051 & 0.7136 & -0.9168 & -0.3844 & $\sim$ \\
\hline \multicolumn{6}{|c|}{$T_{s}=0.3$} \\
\hline$\varepsilon$ & $\overline{\overline{U_{\max }}}$ & $\overline{\overline{U^{\prime}(0)}}$ & 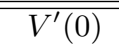 & $\overline{\Theta^{\prime}(0)}$ & $\bar{~} \overline{W(z \rightarrow \infty)}$ \\
\hline-0.75 & 0.3005 & 0.2640 & -0.2545 & -0.3991 & $\sim$ \\
\hline-0.50 & 0.3003 & 0.4187 & -0.4518 & -0.4192 & $\sim$ \\
\hline-0.25 & 0.3001 & 0.5311 & -0.6076 & -0.4309 & $\sim$ \\
\hline 0.00 & 0.3000 & 0.6219 & -0.7358 & -0.4389 & $\sim$ \\
\hline 0.25 & 0.3000 & 0.6997 & -0.8451 & -0.4449 & $\sim$ \\
\hline 0.50 & 0.3000 & 0.7689 & -0.9409 & -0.4496 & $\sim$ \\
\hline 0.75 & 0.3000 & 0.8318 & -1.0265 & -0.4536 & $\sim$ \\
\hline
\end{tabular}

\begin{tabular}{ccccc}
\hline \hline$\varepsilon=-0.75$ & & & \\
\hline \hline$T_{s}$ & $U_{\max }$ & $U^{\prime}(0)$ & $V^{\prime}(0)$ & $\Theta^{\prime}(0)$ \\
0.00 & 0.1884 & 0.2282 & -0.2216 & -0.3279 \\
0.05 & 0.1927 & 0.2288 & -0.2245 & -0.3366 \\
0.10 & 0.2015 & 0.2316 & -0.2287 & -0.3471 \\
0.15 & 0.2153 & 0.2365 & -0.2340 & -0.3588 \\
0.20 & 0.2352 & 0.2436 & -0.2402 & -0.3716 \\
0.25 & 0.2626 & 0.2528 & -0.2471 & -0.3851 \\
0.30 & 0.3005 & 0.2640 & -0.2545 & -0.3991 \\
\hline \hline$\varepsilon=0$ & & & & \\
\hline \hline$T_{s}$ & $U_{\max }$ & $U^{\prime}(0)$ & $V^{\prime}(0)$ & $\Theta^{\prime}(0)$ \\
0.00 & 0.1808 & 0.5102 & -0.6159 & -0.3286 \\
0.05 & 0.1847 & 0.5133 & -0.6269 & -0.3447 \\
0.10 & 0.1937 & 0.5225 & -0.6429 & -0.3621 \\
0.15 & 0.2083 & 0.5381 & -0.6626 & -0.3806 \\
0.20 & 0.2296 & 0.5599 & -0.6851 & -0.3997 \\
0.25 & 0.2592 & 0.5879 & -0.7097 & -0.4192 \\
0.30 & 0.3000 & 0.6219 & -0.7358 & -0.4389 \\
\hline \hline$\varepsilon=0.75$ & & & \\
\hline \hline \multicolumn{6}{c}{$T_{s}$} & $U_{\max }$ & $U^{\prime}(0)$ & $V^{\prime}(0)$ & $\Theta^{\prime}(0)$ \\
0.00 & 0.1779 & 0.6753 & -0.8444 & -0.3135 \\
0.05 & 0.1813 & 0.6791 & -0.8619 & -0.3377 \\
0.10 & 0.1902 & 0.6918 & -0.8867 & -0.3611 \\
0.15 & 0.2051 & 0.7136 & -0.9168 & -0.3844 \\
0.20 & 0.2270 & 0.7443 & -0.9508 & -0.4076 \\
0.25 & 0.2575 & 0.7838 & -0.9878 & -0.4307 \\
0.30 & 0.3000 & 0.8318 & -1.0265 & -0.4536 \\
\hline \hline \multicolumn{7}{c}{}
\end{tabular}

Table 1: Mean-flow data for a range of flow parameters

of the boundary layer seen from increasing $T_{s}$ in Figure 2 (where $\varepsilon=0$ ).

Increasing the values of $\varepsilon$ and $T_{s}$ individually both result in the radial velocity profile, $U(z)$, converging to the free stream boundary condition at a reduced distance from the disk surface. When $T_{s} \neq 0$, we see that increasing $\varepsilon$ results in similar movement of the radial profile towards the disk surface. Likewise, when $\varepsilon \neq 0$, the effect of increasing $T_{s}$ results in the profile maximum moving away from the wall, but the overall convergence of the profile occurs closer to the disk surface.

Similarly, the azimuthal profiles reveal that increasing both $T_{s}$ and $\varepsilon$ produce effects consistent with those observed in the individual cases, i.e. increasing both parameters shifts the $V(z)$ profile towards the disk surface. In summary, the individually observed effects of increasing $\varepsilon$ and $T_{s}$ combine to result in a further narrowing of the boundary layer.

Interestingly, the effect of varying the temperature dependence on the axial and temperature profiles appear to be impacted by the strength of enforced axial flow. When $T_{s}=0.15$, varying $\varepsilon$ has a negligible impact on $W(z)$, whilst at $T_{s}=0.3$ the effect of increasing $\varepsilon$ is the opposite of that seen when $T_{s}=0$, i.e. the outflow acceleration is increased. Similarly, $\Theta(z)$ has a negligible variation with $\varepsilon$ when $T_{s}=0.15$, whilst in the $T_{s}=0.3$ case, the $\Theta(z)$ profile narrows with increasing $\varepsilon$ as opposed to the broadening effect seen when $T_{s}=0$. Recalling the continuity equation, the effects on both $W(z)$ and $\Theta(z)$ can be attributed to the boundary condition for $U(z)$ when $T_{s} \neq 0$.

We see that the radial profile converges to zero when $T_{s}=0$, whilst increasing $\varepsilon$ moves the radial profile towards the disk surface. As such, the area under the $U(z)$ curve decreases with increasing $\varepsilon$, which decreases the magnitude of $W(z)$ throughout the boundary layer. However, when $T_{s} \neq 0$, the radial profile converges to $T_{s}$, which drastically increases the area under $U(z)$ and results in the unbound acceleration observed in $W(z)$ profile. For sufficient axial flow, the narrowing effect of increasing $\varepsilon$ further increases the area under the $U(z)$ curve. Hence, the axial outflow velocity is increased throughout the boundary layer, as can be observed when $T_{s}=0.3$. Due to its dependence on $W(z)$, the $\Theta(z)$ profile is moved towards the disk surface when $T_{s}=0.3$, narrowing the thermal boundary layer (as opposed to the thickening effect seen when varying $\varepsilon$ at $\left.T_{s}=0\right)$.

Summary data for the mean-flow profiles for a range of flow parameters is shown in Table 1. 


\section{Linear convective instability analysis}

In this section we derive the perturbation equations for the linear instability of the flow and proceed to present a full stability analysis under various parameter regimes.

\subsection{Derivation of the perturbation equations}

The mean-flow solutions are now subject to small perturbation quantities leading to

$$
\begin{aligned}
u(r, \theta, z, t) & =\frac{r}{R} U(z)+\hat{u}(r, \theta, z, t), \\
v(r, \theta, z, t) & =\frac{r}{R} V(z)+\hat{v}(r, \theta, z, t), \\
w(r, \theta, z, t) & =\frac{1}{R} W(z)+\hat{w}(r, \theta, z, t), \\
p(r, \theta, z, t) & =\frac{1}{R^{2}} P(z)+\hat{p}(r, \theta, z, t), \\
T(r, \theta, z, t) & =\quad \Theta(z)+\hat{\Theta}(r, \theta, z, t),
\end{aligned}
$$

with the coefficients arising from the non-dimensionalisation process. The governing equations are now non-dimensionalised under new length, velocity, time and pressure scales

$$
\begin{aligned}
& \mathbf{U}^{*}=(u, v, w) r_{s}^{*} \Omega^{*}, \quad\left(r^{*}, z^{*}\right)=(r, z) \delta^{*}, \quad t^{*}=t \frac{L^{*}}{r_{s}^{*} \Omega^{*}}, \\
& p^{*}=p\left(r_{s}^{*} \Omega^{*}\right)^{2} \rho^{*}, \quad T^{*}-T_{\infty}^{*}=T\left(T_{w}^{*}-T_{\infty}^{*}\right), \quad \varepsilon^{*}=\frac{\varepsilon}{T_{w}^{*}-T_{\infty}^{*}} .
\end{aligned}
$$

where $r_{s}^{*}$ is the local radius of the disk at which instability occurs. The local Reynolds number is therefore defined as

$$
R=\frac{r_{s}^{*} \Omega^{*} \delta^{*} \rho^{*}}{\mu_{\infty}^{*}}=\frac{r_{s}^{*}}{\delta^{*}}=r_{s}
$$

which is in interpreted as a non-dimensional radial distance along the disk.

Upon substituting (7) into the newly non-dimensionalised equations, the viscosity function undergoes a Taylor expansion to first order, allowing the mean flow form to be retained.

$$
\mu=\frac{1}{1+\varepsilon(\Theta+\hat{\Theta})} \approx \frac{1}{1+\varepsilon \Theta}\left(1-\frac{\varepsilon \hat{\Theta}}{1+\varepsilon \Theta}\right)=\bar{\mu}+\hat{\mu}
$$

where $\hat{\mu}=-\varepsilon \hat{\Theta} /(1+\varepsilon \Theta)^{2}$. The resulting stability equations are then linearised with respect to perturbation quantities, giving

$$
\begin{aligned}
\frac{\partial \hat{u}}{\partial r}+\frac{\hat{u}}{r}+\frac{1}{r} \frac{\partial \hat{v}}{\partial \theta}+\frac{\partial \hat{w}}{\partial z}= & 0, \\
\mathcal{L} \hat{u}+\frac{r U^{\prime} \hat{w}}{R}+\frac{U \hat{u}}{R}-\frac{2(V+1) \hat{v}}{R}= & -\frac{\partial \hat{p}}{\partial r}+\frac{\bar{\mu}}{R}\left(\nabla^{2} \hat{u}-\frac{2}{r^{2}} \frac{\partial \hat{v}}{\partial \theta}-\frac{\hat{u}}{r^{2}}\right) \\
& +\frac{r U^{\prime \prime} \hat{\mu}}{R^{2}}+\frac{2 U}{R^{2}} \frac{\partial \hat{\mu}}{\partial r}+\frac{\bar{\mu}^{\prime}}{R}\left(\frac{\partial \hat{u}}{\partial z}+\frac{\partial \hat{w}}{\partial r}\right)+\frac{r U^{\prime}}{R^{2}} \frac{\partial \hat{\mu}}{\partial z} \\
\mathcal{L} \hat{v}+\frac{r V^{\prime} \hat{w}}{R}+\frac{U \hat{v}}{R}+\frac{2(V+1) \hat{u}}{R}= & -\frac{1}{r} \frac{\partial \hat{p}}{\partial \theta}+\frac{\bar{\mu}}{R}\left(\nabla^{2} \hat{v}+\frac{2}{r^{2}} \frac{\partial \hat{u}}{\partial \theta}-\frac{\hat{v}}{r^{2}}\right) \\
& +\frac{r V^{\prime \prime} \hat{\mu}}{R^{2}}+\frac{2 U}{r R^{2}} \frac{\partial \hat{\mu}}{\partial \theta}+\frac{\bar{\mu}^{\prime}}{R}\left(\frac{\partial \hat{v}}{\partial z}+\frac{1}{r} \frac{\partial \hat{w}}{\partial \theta}\right)+\frac{r V^{\prime}}{R^{2}} \frac{\partial \hat{\mu}}{\partial z}, \\
\mathcal{L} \hat{w}+\frac{W^{\prime} \hat{w}}{R}= & -\frac{\partial p}{\partial z}+\frac{\bar{\mu}}{R} \nabla^{2} \hat{w} \\
& +\frac{W^{\prime \prime} \hat{\mu}}{R}+\frac{r U^{\prime}}{R^{2}} \frac{\partial \hat{\mu}}{\partial r}+\frac{V^{\prime}}{R^{2}} \frac{\partial \hat{\mu}}{\partial \theta}+\frac{2}{R}\left(\bar{\mu}^{\prime} \frac{\partial w}{\partial z}+W^{\prime} \frac{\partial \hat{\mu}}{\partial z}\right), \\
\mathcal{L} \hat{\Theta}+\Theta^{\prime} \hat{w}= & \frac{1}{R P r} \nabla^{2} \hat{\Theta},
\end{aligned}
$$

where 


$$
\begin{aligned}
\mathcal{L} & =\frac{\partial}{\partial t}+\frac{r U}{R} \frac{\partial}{\partial r}+\frac{V}{R} \frac{\partial}{\partial \theta}+\frac{W}{R} \frac{\partial}{\partial z} \\
\nabla^{2} & =\frac{\partial^{2}}{\partial r^{2}}+\frac{1}{r} \frac{\partial}{\partial r}+\frac{1}{r^{2}} \frac{\partial^{2}}{\partial \theta^{2}}+\frac{\partial^{2}}{\partial z^{2}} .
\end{aligned}
$$

This system is now subject to a parallel-flow approximation, wherein all $r$ coefficients are replaced by $R$. This approximation is representative of a constant boundary-layer thickness across the disk surface. Physically the rotating-disk boundary-layer does not exhibit radial growth and the terminology is borrowed from the approximation's use in growing boundary layers, such as Blasius or Falkner-Skan flows. Following this substitution, the stability equations become separable in $r, \theta$ and $t$, allowing the perturbation quantities to be expressed in normal mode form

$$
(\hat{u}, \hat{v}, \hat{w}, \hat{p}, \hat{\Theta})=(\tilde{u}, \tilde{v}, \tilde{w}, \tilde{p}, \tilde{\Theta}) e^{i(\alpha r+n \theta-\omega t)},
$$

where the variables marked with a tilde are the perturbation eigenfunctions dependent on $z$. Here, $\alpha=$ $\alpha_{r}+i \alpha_{i}$ is the complex radial wavenumber such that $\alpha_{i}<0$ denotes convectively unstable disturbances that grow radially. The azimuthal wavenumber, $n=\beta R$, is an integer quantity representing the number of vortices present on the disk, and $\omega$ is the frequency of the disturbance. Disturbances that rotate with the disk surface are represented by $\omega=0$ in this frame of reference and are henceforth referred to as stationary.

The perturbation quantities in (9) are now expressed in normal mode form and all $\mathcal{O}\left(R^{-2}\right)$ terms are considered negligible.

$$
\begin{aligned}
i \alpha \tilde{u}+\frac{\tilde{u}}{R}+i \beta \tilde{v}+\tilde{w}^{\prime}= & 0, \\
\mathcal{D} \tilde{u}+\frac{W \tilde{u}^{\prime}}{R}+U^{\prime} \tilde{w}+\frac{U \tilde{u}}{R}-\frac{2(V+1) \tilde{v}}{R}= & -i \alpha \tilde{p}+\frac{\bar{\mu}\left(-\alpha^{2} \tilde{u}-\beta^{2} \tilde{u}+\tilde{u}^{\prime \prime}\right)}{R}+\frac{U^{\prime \prime} \tilde{\mu}}{R} \\
& +\frac{\bar{\mu}^{\prime}\left(\tilde{u}^{\prime}+i \alpha \tilde{w}\right)}{R}+\frac{U^{\prime} \tilde{\mu}^{\prime}}{R}, \\
\mathcal{D} \tilde{v}+\frac{W^{\prime} \tilde{v}^{\prime}}{R}+V^{\prime} \tilde{w}+\frac{U \tilde{v}}{R}+\frac{2(V+1) \tilde{u}}{R}= & -i \beta \tilde{p}+\frac{\bar{\mu}\left(-\alpha^{2} \tilde{v}-\beta^{2} \tilde{v}+\tilde{v}^{\prime \prime}\right)}{R}+\frac{V^{\prime \prime} \tilde{\mu}}{R} \\
& +\frac{\bar{\mu}^{\prime}\left(\tilde{v}^{\prime}+i \beta \tilde{w}\right)}{R}+\frac{V^{\prime} \tilde{\mu}^{\prime}}{R}, \\
\mathcal{D} \tilde{w}+\frac{W \tilde{w}^{\prime}}{R}+\frac{W^{\prime} \tilde{w}}{R}= & -\tilde{p}^{\prime}+\frac{\bar{\mu}\left(-\alpha^{2} \tilde{w}-\beta^{2} \tilde{w}+\tilde{w}^{\prime \prime}\right)}{R}+\frac{W^{\prime \prime} \tilde{\mu}}{R} \\
& +\frac{i \tilde{\mu}\left(\alpha U^{\prime}+\beta V^{\prime}\right)}{R}+\frac{2\left(\bar{\mu}^{\prime} \tilde{w}^{\prime}+W^{\prime} \tilde{\mu}^{\prime}\right)}{R}, \\
-i \omega \tilde{\Theta}+i \alpha U \tilde{\Theta}+i \beta V \tilde{\Theta}+\frac{W \tilde{\Theta}^{\prime}}{R}+\Theta^{\prime} \tilde{w}= & \frac{\left(-\alpha^{2} \tilde{\Theta}-\beta^{2} \tilde{\Theta}+\tilde{\Theta}^{\prime \prime}\right)}{R \operatorname{Pr}},
\end{aligned}
$$

where $\mathcal{D}=i(-\omega+\alpha U+\beta V)$, and $\tilde{\mu}=-\varepsilon \tilde{\Theta} /(1+\varepsilon \Theta)^{2}$. System (10) states the governing perturbation equations as a quadratic eigenvalue problem of the form $A_{2} \alpha^{2}+A_{1} \alpha+A_{0}=\tilde{Q}$, where $\tilde{Q}=(\tilde{u}, \tilde{v}, \tilde{w}, \tilde{p}, \tilde{\Theta})^{\mathrm{T}}$ is the vector of eigenfunctions, and the quantities $A_{j}$ are matrices containing the coefficients of the $\mathcal{O}\left(\alpha^{j}\right)$ terms. The eigenfunctions are then computed according to the boundary conditions that constrain the perturbations to reside within the boundary layer

$$
\begin{array}{r}
\tilde{u}(y=0)=\tilde{v}(y=0)=\tilde{w}(y=0)=\tilde{w}^{\prime}(y=0)=\tilde{\Theta}(y=0)=0, \\
\tilde{u}(y \rightarrow \infty) \rightarrow \tilde{v}(y \rightarrow \infty) \rightarrow \tilde{w}(y \rightarrow \infty) \rightarrow \tilde{p}(y \rightarrow \infty) \rightarrow \tilde{\Theta}(y \rightarrow \infty) \rightarrow 0 .
\end{array}
$$

The neutral temporal and spatial stability solutions are obtained via a Chebyshev polynomial discretisation method. An exponential map is used to transform the Gauss-Lobatto collocation points into the physical domain. The stability equations are then solved as primitive variables over 100 collocation points distributed between the upper and lower boundaries, with the exception of the conditions described in (11) which are imposed at $z=0$ and $z=z_{\max }$, where suitable mean-flow convergence is again found at $z_{\max }=20$. Further details of the numerical method employed here can be found in Alveroğlu [1] and the code used here is based on that developed by Alveroğlu during that related work. 


\subsection{Neutral stability curves}

We now proceed to compute the neutral stability curves for various parameter combinations of the mean flow. In all cases, the stability of the flow will be examined by plotting neutral points $\left(\alpha_{i}=0\right)$ in the $(R, n)$ - and $(R, \psi)$-planes, where $\psi=\tan ^{-1}\left(\beta / \alpha_{r}\right)$ is the orientation angle of spiral vortices relative to a circle concentric to the disk. Both $n$ and $\psi$ are physical, measurable quantities. Whilst $n$ must be interpreted as an integer, it is permitted to take any value during the mathematical analysis.

All neutrals curves obtained are found to have a two-lobed structure: the upper representing the type I crossflow instability and the lower representing the type II viscous instability. This is consistent with all previous work on the rotating disk boundary layer, as discussed in $\S 1$. Our discussions focus on the critical Reynolds number, $R_{c}$, which is the lowest Reynolds number that permits an unstable mode.

\subsubsection{Temperature-dependent viscosity (no axial flow)}
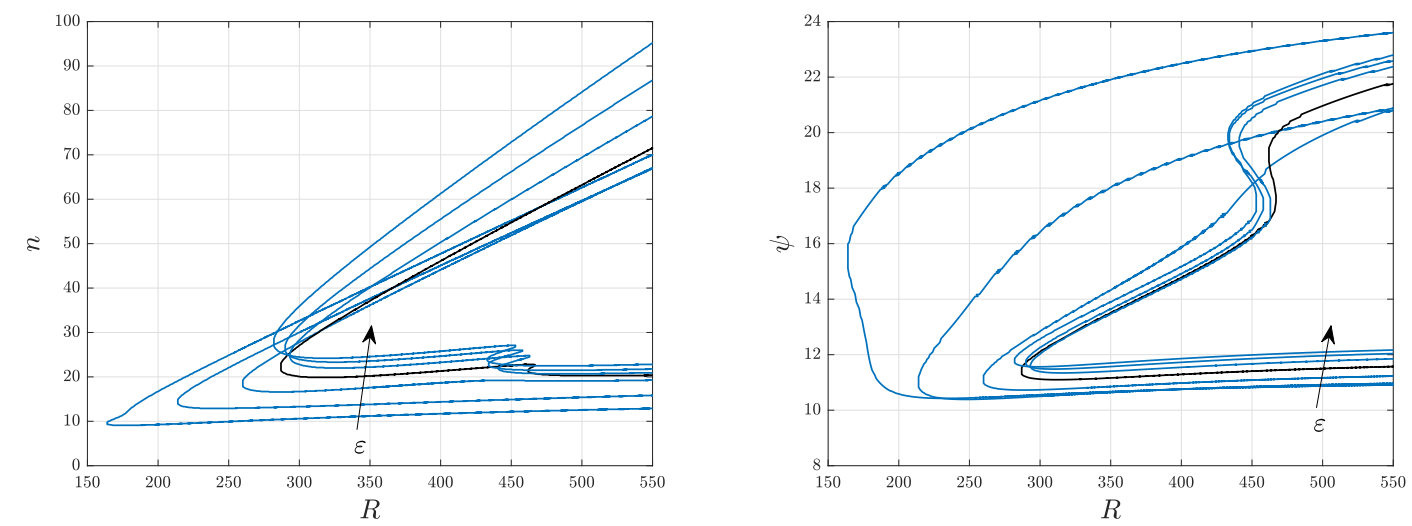

Figure 4: Vortex-number and -angle neutral stability curves for the range $-0.75 \leq \varepsilon \leq 0.75$ in increments of 0.25 .

Figure 4 shows neutral stability curves for stationary disturbances $(\omega=0)$ for a range of temperature dependencies. We see that $\varepsilon=-0.75$ is the most unstable case for both modes by a significant margin. Examining the type I mode, we find that increasing the temperature dependence from $\varepsilon=-0.75$ results in a sharp increase in the critical Reynolds number. This stabilising behaviour continues through the gas-type regime, to the temperature independent case, and into the liquid-type regime to $\varepsilon=0.25$. However, a further increase in the value of $\varepsilon$ results in a reduction in the critical Reynolds number, such that the case when $\varepsilon=0.75$ is in fact less stable than the temperature independent case. It can therefore be inferred that a turning point in the stabilising behaviour of the type I mode exists in the range $0 \leq \varepsilon \leq 0.5$.

The type II mode exhibits similar behaviour, although it appears that the maximum of the type II critical Reynolds number occurs when $\varepsilon=0$. It is possible that any temperature dependence leads to destabilisation of the type II mode. The type II mode is also significantly less stable for cases when $\varepsilon<0$, such that when $\varepsilon=-0.5$, the two modes cannot be visually distinguished, whilst for the case when $\varepsilon=-0.75$, the type II mode is dominant.

In spite of its non-monotonic relationship with the critical Reynolds number, increasing $\varepsilon$ appears to consistently increase the critical number of vortices $n_{c}$, as well as the range of unstable $n$ values. We see that the critical wave angles for both modes follow the same trends with changing $\varepsilon$; with the $\varepsilon=-0.75$ neutral curve encompassing the largest range of unstable wave angles.

\subsubsection{Axial flow (no temperature dependence)}

Figure 5 shows the stationary mode neutral stability curves for a range of axial flow strengths for the case when $\varepsilon=0$. As $T_{s}$ is increased, the critical Reynolds number is significantly increased, stabilising the type I mode. The value of $n_{c}$ also increases significantly, as well as the range of unstable values of $n$ enclosed by the successive curves. Conversely, the type II mode is initially destabilised for small values of $T_{s}$, before steadily stabilising as $T_{s}$ is increased further. From this it can be inferred that there is a minimum stability of the type II mode in the region $0<T_{s}<0.1$.

For larger values of $T_{s}$, though both modes are shifted along the $R$-axis, the type II mode becomes more prominent as the type I mode undergoes greater stabilisation. It is likely that further increasing $T_{s}$ will lead to the type II mode becoming the dominant form of instability. 



Figure 5: Vortex-number and -angle neutral stability curves for the range $0 \leq T_{s} \leq 0.3$ in increments of 0.05 .

As might be expected, the behaviour of the critical wave angle follows that of $n_{c}$. The range of unstable wave angles is decreased as $T_{s}$ is increased, though the unstable angles enclosed by the curves are significantly increased.

\subsubsection{Combined effects}

Figure 6 depicts the neutral curves for a range of temperature dependences at a constant, non-zero enforced axial flow and vice versa. We see that the effect of increasing $\varepsilon$ is consistent with that seen in Figure 4, where increasing $\varepsilon$ results in increased flow stability limited to some positive value of $\varepsilon$, beyond which flows are less stable. At $T_{s}=0.15$, the turning point for stabilising behaviour of increasing $\varepsilon$ appears in the range $0 \leq \varepsilon \leq 0.5$, which is consistent with the unenforced case (i.e. $T_{s}=0$ ). When $T_{s}=0.3$, the stabilising effect is extended and the point of maximum stability occurs in the range $0.25 \leq \varepsilon \leq 0.75$. The wave angles enclosed by the neutral curves increase significantly for all $\varepsilon$ values when $T_{s}=0.15$, increasing further still when $T_{s}=0.3$.

Examining the type II mode behaviour, the lower branch critical Reynolds number increases as $\varepsilon$ is increased; an effect consistent with that seen in Figure 4 for the range $\varepsilon \leq 0$. Examining the individual curves in this range shows that a flow with $\varepsilon=-0.5$ is type I dominant when $T_{s}=0.15$ and $T_{s}=0.3$, unlike for the case when $T_{s}=0$ where there is no obvious distinction between the branches. However, for the cases when $\varepsilon>0$, there does not appear to be an immediately discernible relationship between the type II critical Reynolds number and value of $\varepsilon$.

Increasing $T_{s}$ yields effects consistent with that seen in Figure 5, in that both modes are stabilised (the type I mode more significantly), the range of vortex values enclosed by the neutral curves increases and the enclosed range of wave angles decreases.

When $\varepsilon=-0.75$, we find that the curves are significantly less stable than those in Figure 5 and that the type II mode is dominant for all $T_{s}$ values. As $T_{s}$ is increased, the type I mode is appreciatively stabilised. This further enforces the dominance of the type II mode caused by the temperature dependence (in spite of it also being stabilised).

When $\varepsilon=0.75$, we observe neutral curves very similar to those of the temperature-independent case presented in Figure 5. The curves for values of $T_{s} \geq 0.1$ are slightly stabilised for this value of temperature dependence, whilst the cases when $T_{s}=0$, and $T_{s}=0.05$, both yield a reduced critical Reynolds number when compared to the temperature-independent problem.

Figure 7 plots a range of $\varepsilon$ values against their associated type I critical Reynolds numbers at various $T_{s}$ values; each curve therefore represents a different fixed axial flow strength. Note that the range of $\varepsilon$ values reported on is extended beyond that used for the neutral curves. In each case an optimal value of $\varepsilon>0$ (with respect to the maximum value of the critical Reynolds number) is determined such that further increases beyond this point result in flows that are progressively less stable. We find that the most stable flow is produced for an increasingly greater value of $\varepsilon$ as $T_{s}$ is increased. Furthermore, the range of $\varepsilon$ values that results in flows that are more stable than the temperature independent case is increased with $T_{s}$. Critical data for the range of $\varepsilon$ and $T_{s}$ values considered is presented here in Table 2.

\subsection{Growth rates and $e^{N}$ analysis}

Using an $e^{N}$ type analysis, as outlined by van Ingen [18], we are able to investigate the evolution of the complex radial wave number for a range of axial flow strengths and temperature dependencies. First, the 

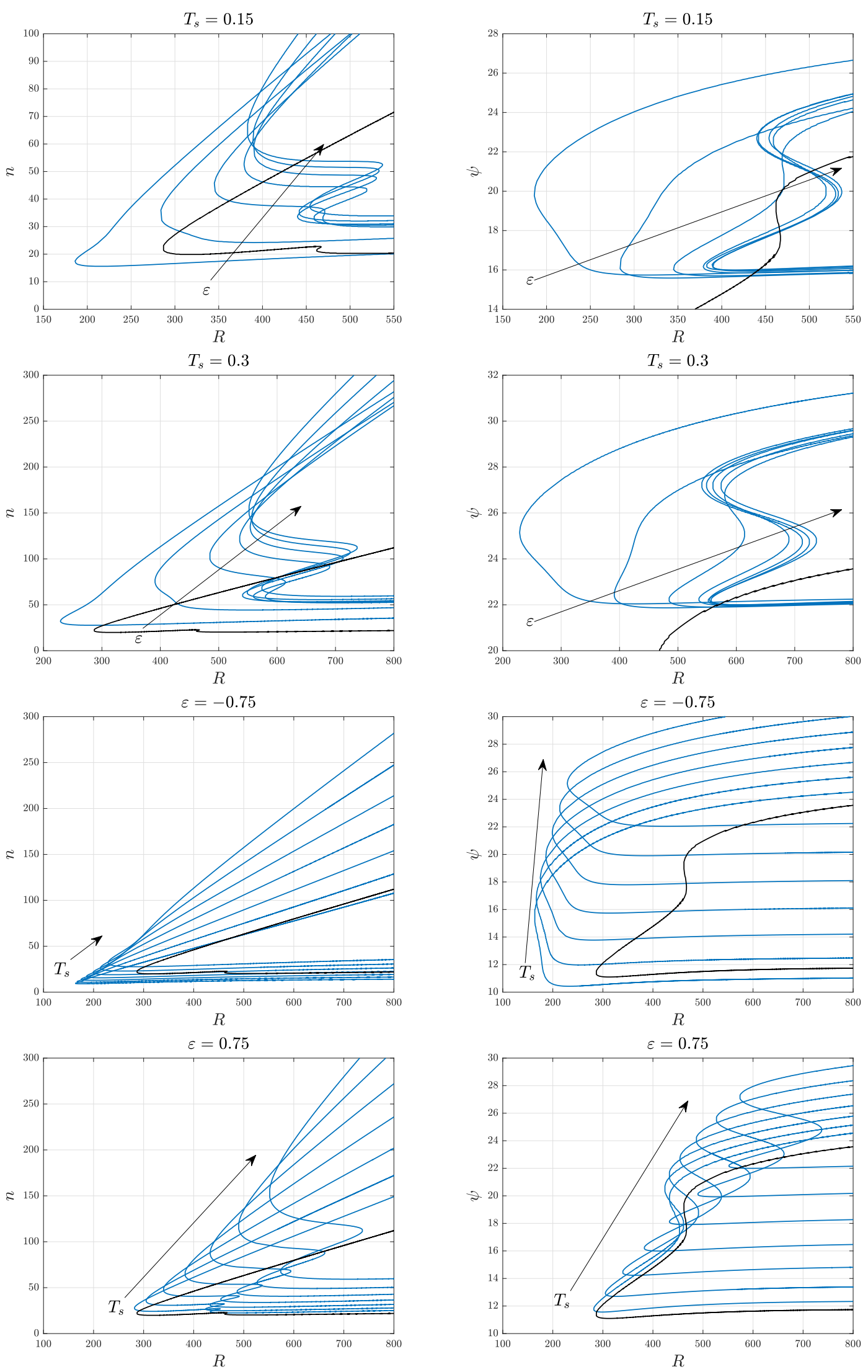

Figure 6: Vortex-number and -angle neutral stability curves for a range of axial flow strengths and temperature dependencies. Curves show $-0.75 \leq \varepsilon \leq 0.75$ in increments of 0.25 , for the indicated $T_{s}$ value or $0 \leq T_{s} \leq 0.3$ in increments of 0.05 , for the indicated $\varepsilon$ value. The black curves are provided as a reference to the temperature independent, zero axial enforcement $\left(\varepsilon=T_{s}=0\right)$ case. 


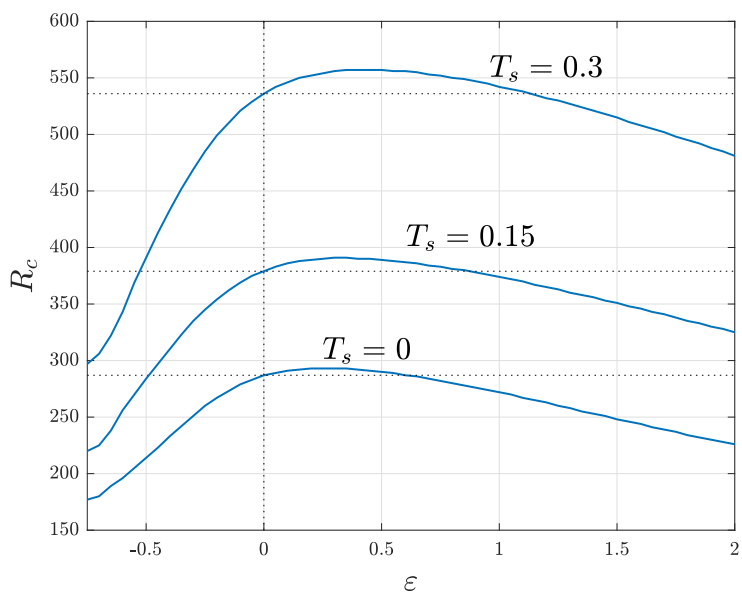

Figure 7: Plot of the type I critical Reynolds number arising from each $T_{s}=0,0.15 \& 0.3$. The dotted lines indicate a reference for the critical Reynolds number associated with the temperature independent case (i.e. $\varepsilon=0)$ at each $T_{s}$.

\begin{tabular}{|c|c|c|c|c|c|c|c|c|}
\hline \multicolumn{9}{|c|}{$T_{S}=0$} \\
\hline$\varepsilon$ & $R_{c}$ & $n_{c}$ & $\alpha_{r, c}$ & $\psi_{c}$ & $\left(R_{c}\right)$ & $\left(n_{c}\right)$ & $\left(\alpha_{r, c}\right)$ & $\left(\psi_{c}\right)$ \\
\hline-0.75 & $\sim$ & $\sim$ & $\sim$ & $\sim$ & 164 & 10 & 0.2096 & 15.55 \\
\hline-0.5 & 214 & 15 & 0.3450 & 11.02 & $\sim$ & $\sim$ & $\sim$ & $\sim$ \\
\hline-0.25 & 260 & 19 & 0.3624 & 11.11 & $\sim$ & $\sim$ & $\sim$ & $\sim$ \\
\hline 0 & 287 & 23 & 0.3842 & 11.41 & 462 & 22 & 0.1325 & 19.20 \\
\hline 0.25 & 293 & 25 & 0.4114 & 11.60 & 441 & 22 & 0.1390 & 19.66 \\
\hline 0.5 & 290 & 27 & 0.4404 & 11.72 & 434 & 23 & 0.1462 & 19.76 \\
\hline 0.75 & 282 & 28 & 0.4718 & 11.78 & 433 & 24 & 0.1526 & 19.85 \\
\hline \multicolumn{9}{|c|}{$T_{S}=0.15$} \\
\hline$\varepsilon$ & $R_{c}$ & $n_{c}$ & $\alpha_{r, c}$ & $\psi_{c}$ & $\left(R_{c}\right)$ & $\left(n_{c}\right)$ & $\left(\alpha_{r, c}\right)$ & $\left(\psi_{c}\right)$ \\
\hline-0.75 & $\sim$ & $\sim$ & $\sim$ & $\sim$ & 186 & 18 & 0.2617 & 19.77 \\
\hline-0.5 & 284 & 36 & 0.4402 & 16.06 & $\sim$ & $\sim$ & $\sim$ & $\sim$ \\
\hline-0.25 & 345 & 46 & 0.4624 & 16.02 & 469 & 33 & 0.1772 & 21.61 \\
\hline 0 & 379 & 53 & 0.4793 & 16.16 & 459 & 34 & 0.1739 & 22.56 \\
\hline 0.25 & 390 & 60 & 0.5267 & 16.16 & 440 & 34 & 0.1830 & 22.68 \\
\hline 0.5 & 389 & 61 & 0.5327 & 16.28 & 442 & 36 & 0.1905 & 22.69 \\
\hline 0.75 & 383 & 66 & 0.5850 & 16.21 & 454 & 38 & 0.1970 & 22.69 \\
\hline \multicolumn{9}{|c|}{$T_{S}=0.3$} \\
\hline 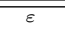 & $R_{c}$ & $n_{c}$ & $\bar{\alpha} \alpha_{r, c}$ & $\psi_{c}$ & $\left(R_{c}\right)$ & $\left(n_{c}\right)$ & $\left(\alpha_{r, c}\right)$ & $\left(\psi_{c}\right)$ \\
\hline-0.75 & $\sim$ & $\sim$ & $\sim$ & $\sim$ & 229 & 33 & 0.2991 & 25.15 \\
\hline-0.5 & 391 & 76 & 0.4692 & 22.45 & $\sim$ & $\sim$ & $\sim$ & $\sim$ \\
\hline-0.25 & 485 & 103 & 0.5186 & 22.23 & 580 & 59 & 0.2011 & 26.79 \\
\hline 0 & 536 & 122 & 0.5557 & 22.23 & 560 & 60 & 0.2060 & 27.20 \\
\hline 0.25 & 554 & 135 & 0.5950 & 22.23 & 541 & 61 & 0.2165 & 27.22 \\
\hline 0.5 & 557 & 145 & 0.6365 & 22.21 & 549 & 64 & 0.2241 & 27.20 \\
\hline 0.75 & 552 & 153 & 0.6800 & 22.18 & 574 & 69 & 0.2309 & 27.18 \\
\hline
\end{tabular}

\begin{tabular}{ccccccccc}
\hline \hline \multicolumn{1}{c}{$\varepsilon=-0.75$} & & & & & & & \\
\hline \hline$T_{s}$ & $R_{c}$ & $n_{c}$ & $\alpha_{r, c}$ & $\psi_{c}$ & $\left(R_{c}\right)$ & $\left(n_{c}\right)$ & $\left(\alpha_{r, c}\right)$ & $\left(\psi_{c}\right)$ \\
0 & $\sim$ & $\sim$ & $\sim$ & $\sim$ & 164 & 10 & 0.2096 & 15.52 \\
0.05 & $\sim$ & $\sim$ & $\sim$ & $\sim$ & 167 & 12 & 0.2275 & 16.77 \\
0.1 & $\sim$ & $\sim$ & $\sim$ & $\sim$ & 175 & 14 & 0.2409 & 18.31 \\
0.15 & $\sim$ & $\sim$ & $\sim$ & $\sim$ & 186 & 18 & 0.2617 & 19.77 \\
0.2 & $\sim$ & $\sim$ & $\sim$ & $\sim$ & 199 & 22 & 0.2721 & 21.57 \\
0.25 & $\sim$ & $\sim$ & $\sim$ & $\sim$ & 214 & 27 & 0.2854 & 23.34 \\
0.3 & $\sim$ & $\sim$ & $\sim$ & $\sim$ & 229 & 33 & 0.2991 & 25.15 \\
\hline \hline$\varepsilon=0$ & & & & & & & & \\
\hline \hline$T_{s}$ & $R_{c}$ & $n_{c}$ & $\alpha_{r, c}$ & $\psi_{c}$ & $\left(R_{c}\right)$ & $\left(n_{c}\right)$ & $\left(\alpha_{r, c}\right)$ & $\left(\psi_{c}\right)$ \\
0 & 287 & 23 & 0.3842 & 11.41 & 462 & 22 & 0.1325 & 19.20 \\
0.05 & 308 & 29 & 0.4175 & 12.71 & 441 & 24 & 0.1470 & 20.17 \\
0.1 & 339 & 39 & 0.4490 & 14.34 & 443 & 28 & 0.1609 & 21.28 \\
0.15 & 379 & 53 & 0.4793 & 16.16 & 459 & 34 & 0.1739 & 22.56 \\
0.2 & 427 & 71 & 0.5073 & 18.13 & 485 & 41 & 0.1858 & 23.99 \\
0.25 & 479 & 94 & 0.5326 & 20.16 & 519 & 49 & 0.1965 & 25.55 \\
0.3 & 536 & 122 & 0.5557 & 22.23 & 560 & 60 & 0.2060 & 27.20 \\
\hline \hline$\varepsilon=0.75$ & & & & & & & \\
\hline \hline$T$ & & & & & & & & \\
0 & $R_{c}$ & $n_{c}$ & $\alpha_{r, c}$ & $\psi_{c}$ & $\left(R_{c}\right)$ & $\left(n_{c}\right)$ & $\left(\alpha_{r, c}\right)$ & $\left(\psi_{c}\right)$ \\
0.05 & 382 & 28 & 0.4718 & 11.78 & 433 & 24 & 0.1526 & 19.85 \\
0.1 & 340 & 48 & 0.5093 & 12.92 & 424 & 27 & 0.1683 & 20.52 \\
0.15 & 383 & 66 & 0.5475 & 14.46 & 433 & 32 & 0.1829 & 21.51 \\
0.2 & 433 & 88 & 0.6194 & 16.21 & 454 & 38 & 0.1970 & 22.69 \\
0.25 & 490 & 117 & 0.6512 & 18.12 & 487 & 46 & 0.2092 & 24.07 \\
0.3 & 552 & 153 & 0.6800 & 22.12 & 527 & 56 & 0.2207 & 25.57 \\
\hline \hline
\end{tabular}

Table 2: Critical data for a range of $\varepsilon$ values at fixed $T_{s}$ values and vice versa. Type II critical data is indicated by bracketed headings. Values marked by $\sim$ are ommitted due to the indefinite division between each branch.

amplitude of a disturbance at radial positions $r$ and $r+\mathrm{d} r$ are considered.

$$
\begin{aligned}
a(r)=|(\hat{u}, \hat{v}, \hat{w}, \hat{p}, \hat{\Theta})| & =|(\tilde{u}, \tilde{v}, \tilde{w}, \tilde{p}, \tilde{\Theta})| e^{-\alpha_{i} r} \\
a(r+\mathrm{d} r) & =|(\tilde{u}, \tilde{v}, \tilde{w}, \tilde{p}, \tilde{\Theta})| e^{-\alpha_{i}(r+\mathrm{d} r)}
\end{aligned}
$$

This leads to the ratio of amplitudes

$$
\frac{a(r+\mathrm{d} r)}{a(r)}=e^{-\alpha_{i} \mathrm{~d} r}
$$

Recalling the definition of the Reynolds number (8), we replace $r$ with $R$ and integrate from some initial position $R_{0}$ (interpreted as the smallest Reynolds number, at a fixed value of $n$, for which instability is observed) to the location under consideration, $R$. This leads to the amplitude ratio

$$
N=\ln \left(\frac{a}{a_{0}}\right)=-\int_{R_{0}}^{R} \alpha_{i} \mathrm{~d} R
$$

This quantity can be computed for a range of fixed vortex numbers $n$ from which an enveloping curve can be fitted. The enveloping curve can then be used to approximate a transition region by comparison to empirical transition data. Cooper \& Carpenter [5] utilise this method for transition prediction of the flow over a rotating disk with a compliant surface and the in-depth review by van Ingen [18] highlights a number of comparisons between $e^{N}$ predictions and stability experiments. Malik et al. [30] determined that $N=10.7$ according to a transitional Reynolds number of 513, later updating this to be in the region $9 \leq N \leq 10$ [35]. 
It is noted here that modifications to the flow may result in a variation of the transitional $N$-factor, though for the purposes of this qualitative study, $N=9$ will be utilised as the reference amplitude ratio required for the prediction of transitional flows.

Figure 8 displays the enveloping $N$-curve formed from a range of amplitude ratios from the temperature independent, zero enforced flow case (i.e. $\varepsilon=T_{s}=0$ ). Here, the curve crosses at $N=9$ when $R \approx 521$, showing good agreement with the results presented by Wilkinson \& Malik [35]. Figure 9 shows the $N$ curves for a range of temperature dependencies and axial flow strengths. It can be seen that by varying $\varepsilon$ for fixed $T_{s}$, the temperature independent case has the largest transitional Reynolds number. It is likely that this behaviour mimics that of the neutral curves, where a maximum transitional Reynolds number exists. Interestingly, for the case when $T_{s}=0.3$, the transitional Reynolds number for flows with $\varepsilon=0.75$ is lower than the temperature-independent case, in spite of the latter having a larger $R_{c}$ than the former. This indicates that the relationship between the critical and transitional Reynolds numbers is not strictly monotonic.

We note that the gradient of the $N$-curve is reduced with increasing $T_{s}$. This suggests that a stronger axial flow reduces the disturbance growth rate and delays the onset of transition. This effect is consistent for all $\varepsilon$ values considered and is most likely the reason for the previously discussed increase in transitional Reynolds number in spite of a reduced critical Reynolds number. Additional $N$-curve data is presented in Table 3.

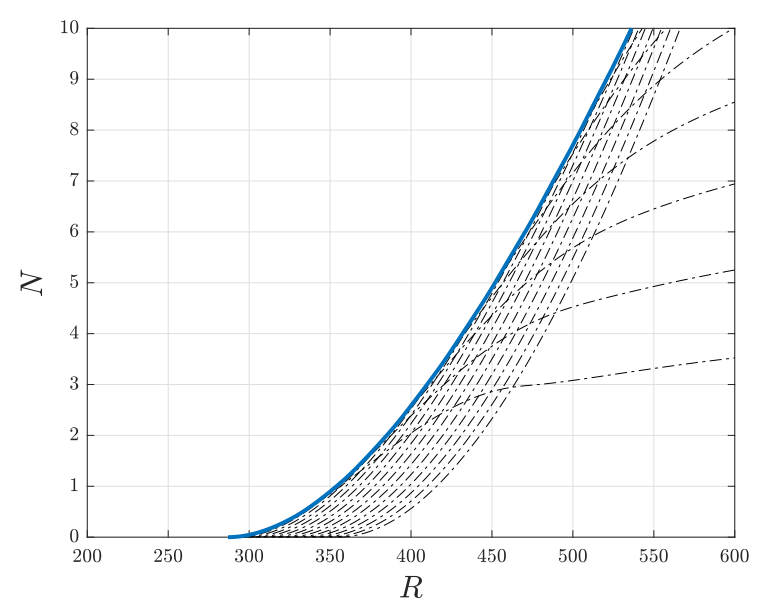

Figure 8: Amplitude ratio curve for $\varepsilon=T_{s}=0$. The dashed curves (--) represent amplitude ratio curves at fixed $n$; the solid curve is the enveloping $N$-factor curve used to determine a transitional Reynolds number.

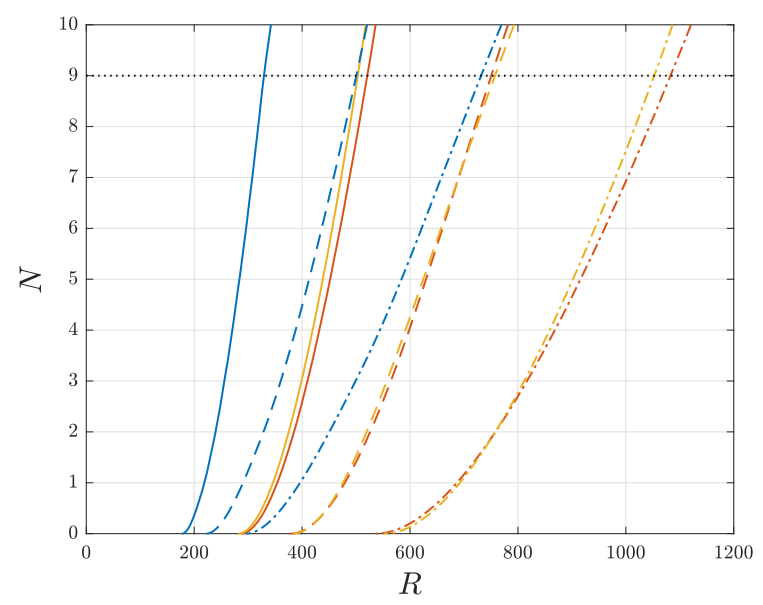

Figure 9: Amplitude ratio curves for a range of $\varepsilon$ and $T_{s}$ values. Solid lines $(-)$ represent $T_{s}=0$; dashed lines $(--)$ represent $T_{s}=0.15$; dash-dot lines $(-\cdot)$ represent $T_{s}=0.3$. Line colours blue, red and yellow represent $\varepsilon=-0.75,0$ and 0.75 respectively. The black, dotted line $(\cdots)$ indicates $N=9$ i.e. the transitional $N$-factor. 


\begin{tabular}{ccc}
\hline \hline$T_{s}$ & $\varepsilon$ & $R(N=9)$ \\
0 & -0.75 & 329 \\
0 & 0 & 521 \\
0 & 0.75 & 503 \\
0.15 & -0.75 & 500 \\
0.15 & 0 & 751 \\
0.15 & 0.75 & 758 \\
0.3 & -0.75 & 732 \\
0.3 & 0 & 1083 \\
0.3 & 0.75 & 1053 \\
\hline \hline
\end{tabular}

Table 3: Transitional Reynolds numbers, $R(N=9)$, for a range of temperature dependencies and axial flow strengths.

\subsection{Eigenfunctions}

It is useful to examine the type I eigenfunctions which will be used to conduct an energy balance analysis in the following section. Here all eigenfunctions are assessed at $R=R_{c}+200$ (i.e. well into the unstable regime), and the value of $\alpha$ chosen such that the most amplified disturbance, at this particular Reynolds number, is selected.

The plots depicted in Figure 10 are the magnitudes of the perturbation eigenfunctions for $\varepsilon$ in the range $-0.75 \leq \varepsilon \leq 2$. We see that, as $\varepsilon$ is increased, the eigenfunction profiles are narrowed. This effect can largely be attributed to a mirroring of the mean-flow profiles. A notable exception is seen where the $|\tilde{\Theta}(z)|$ profile narrows as $\varepsilon$ is increased, as opposed to the broadening of the mean-flow profile depicted in Figure 1 . We further notice that for positive values of $\varepsilon$, the two maxima of the $|\tilde{u}(z)|$ profile become more prominent as $\varepsilon$ is increased and occur closer to the disk surface. Increasing $\varepsilon$ in the range $-0.75 \leq \varepsilon \leq-0.5$ decreases the maximum of the $|\tilde{v}(z)|$ profile. Increasing $\varepsilon$ beyond this leads to an increase in the maximum of the $|\tilde{v}(z)|$ profile. Similarly, increasing $\varepsilon$ initially also decreases the maximum of the $|\tilde{w}(z)|$ profile. The limit of this behaviour occurs in the range $0 \leq \varepsilon \leq 0.5$, where an increase in the temperature dependence parameter thereafter results in the profile maximum increasing.

Figure 11 depicts the magnitudes of the perturbation eigenfunctions for a range of enforced axial flow strengths. We see that the velocity perturbation profiles narrow as $T_{s}$ is increased, decaying closer to the disk surface. Again, this can be seen as a mirroring of the mean-flow profiles in Figure 2. For small values of $T_{s}$, the two maxima of the radial eigenfunction $|\tilde{u}(z)|$ are both reduced. The maximum located further from the disk surface continues to decrease for greater values of $T_{s}$, eventually becoming entirely suppressed in the case when $T_{s}=0.3$. It is inferred that this is a direct response to the suppression of the mean radial velocity inflection seen in Figure 2. We also observe that the maximum of the azimuthal eigenfunction is increased as the axial flow strength is increased, whereas the maximum of the $|\tilde{w}(z)|$ profile is reduced.

Although not shown here, the plots for the combined effects of temperature dependence and enforced axial flow on the perturbation eigenfunctions are such that increasing $\varepsilon$ produces a narrowing effect consistent with that seen in Figure 10 and this effect is strengthened by the presence of enforced axial flow. Enforced axial flow also acts to amplify the primary maximum of the $|\tilde{u}(z)|$ profile, as well as the maxima of both the $|\tilde{v}(z)|$ and $|\tilde{\Theta}(z)|$ profiles. Conversely, the maximum of the axial profile is significantly reduced for all $\varepsilon$ values considered here. Other effects such as the variation in profile maxima with increasing $\varepsilon$ are largely consistent with the $T_{s}=0$ case. Similarly, the effect of increasing $T_{s}$ is largely consistent with the $\varepsilon=0$ case. The primary maximum of the $|\tilde{u}(z)|$ profile and the maxima of the $|\tilde{v}(z)|,|\tilde{w}(z)|$ and $|\tilde{\Theta}(z)|$ profiles are all increased for all $T_{s}$ values considered.

\subsection{Energy balance analysis}

It is possible to conduct an analysis of the energy balance within the system to better understand the underlying mechanisms of the flow instability. Following Cooper \& Carpenter [5], an energy balance equation is now derived from the eigenfunctions to examine the energetic input and output of a disturbance to the mean flow.

To begin, the momentum stability equations (9b)-(9d) are multiplied by their corresponding velocity 

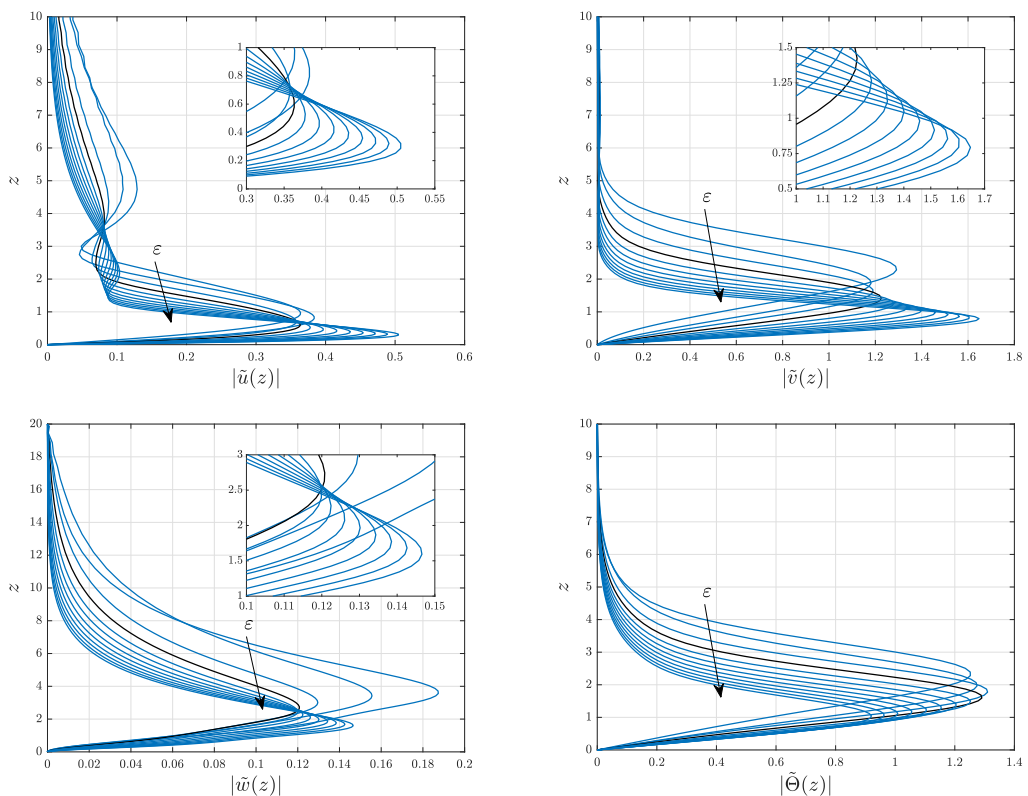

Figure 10: Type I eigenfunctions for $-0.75 \leq \varepsilon \leq 2$ with 0.25 increments. The black curve on each plot is provided as reference to the case of $\varepsilon=T_{s}=0$.
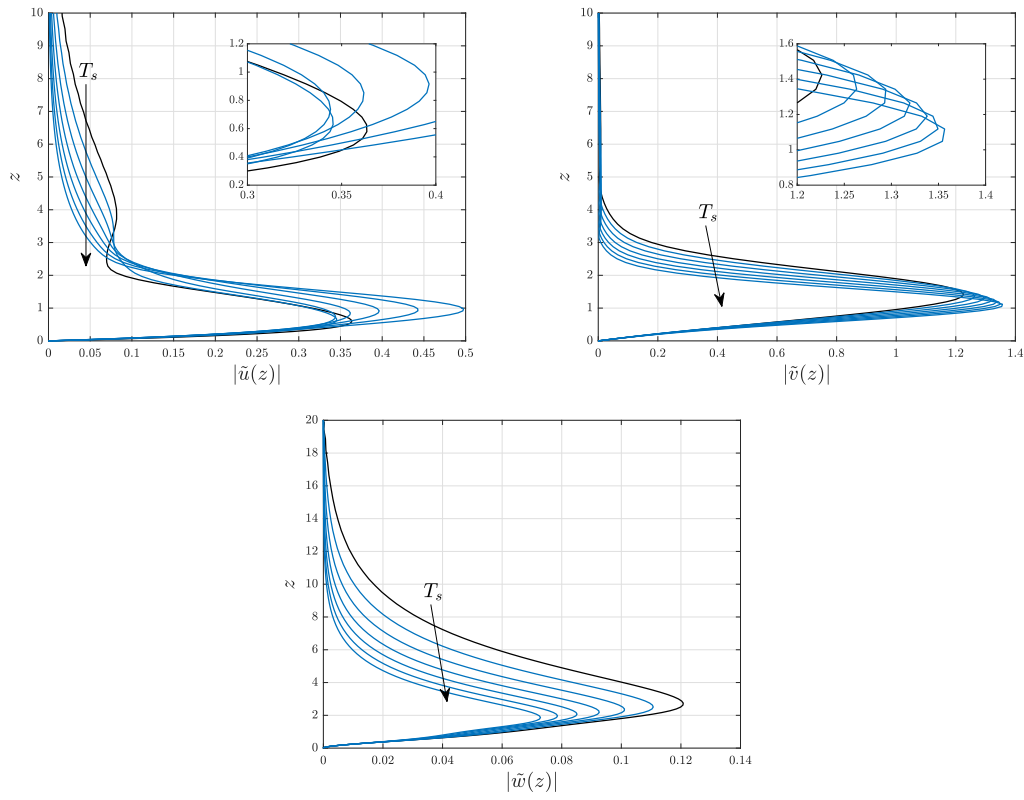

Figure 11: Type I eigenfunctions for $0 \leq T_{s} \leq 0.3$ with 0.05 increments. The black curve on each plot is provided as reference to the case of $\varepsilon=T_{s}=0$. 
component and summed

$$
\begin{aligned}
& \hat{u} \frac{\partial \hat{u}}{\partial t}+\hat{v} \frac{\partial \hat{v}}{\partial t}+\hat{w} \frac{\partial \hat{w}}{\partial t}+U\left(\hat{u} \frac{\partial \hat{u}}{\partial r}+\hat{v} \frac{\partial \hat{v}}{\partial r}+\hat{w} \frac{\partial \hat{w}}{\partial r}\right)+\frac{V}{R}\left(\hat{u} \frac{\partial \hat{u}}{\partial \theta}+\hat{v} \frac{\partial \hat{v}}{\partial \theta}+\hat{w} \frac{\partial \hat{w}}{\partial \theta}\right)+\frac{W}{R}\left(\hat{u} \frac{\partial \hat{u}}{\partial z}+\hat{v} \frac{\partial \hat{v}}{\partial z}+\hat{w} \frac{\partial \hat{w}}{\partial z}\right) \\
& +\frac{U\left(\hat{u}^{2}+\hat{v}^{2}\right)}{R}+U^{\prime} \hat{u} \hat{w}+V^{\prime} \hat{v} \hat{w}+\frac{W^{\prime} \hat{w}^{2}}{R}=-\left(\hat{u} \frac{\partial \hat{p}}{\partial r}+\hat{v} \frac{\partial \hat{p}}{\partial \theta}+\hat{w} \frac{\partial \hat{p}}{\partial z}\right)+\frac{\bar{\mu}}{R}\left(\hat{u}_{j} \frac{\partial \sigma_{i j}}{\partial x_{i}}\right) \\
& +\frac{\bar{\mu}^{\prime}}{R}\left[\hat{u}\left(\frac{\partial \hat{w}}{\partial r}+\frac{\partial \hat{u}}{\partial z}\right)+\hat{v}\left(\frac{1}{R} \frac{\partial \hat{w}}{\partial \theta}+\frac{\partial \hat{v}}{\partial z}\right)+2 \hat{w} \frac{\partial \hat{w}}{\partial z}\right]+\frac{1}{R}\left[\hat{u} \frac{\partial\left(U^{\prime} \hat{\mu}\right)}{\partial z}+\hat{v} \frac{\partial\left(V^{\prime} \hat{\mu}\right)}{\partial z}+\hat{w} \frac{\partial\left(W^{\prime} \hat{\mu}\right)}{\partial z}\right] \\
& +\frac{\hat{w}}{R}\left(U^{\prime} \frac{\partial \hat{\mu}}{\partial r}+\frac{V^{\prime}}{R} \frac{\partial \hat{\mu}}{\partial \theta}+W^{\prime} \frac{\partial \hat{\mu}}{\partial z}\right) .
\end{aligned}
$$

Here $\sigma_{i j}$ represents the viscous stress terms

$$
\begin{aligned}
& \sigma_{11}=\frac{\partial \hat{u}}{\partial r} \quad \sigma_{12}=\frac{1}{R} \frac{\partial \hat{u}}{\partial \theta} \quad \sigma_{13}=\frac{\partial \hat{u}}{\partial z}, \\
& \sigma_{21}=\frac{\partial \hat{v}}{\partial r} \quad \sigma_{22}=\frac{1}{R} \frac{\partial \hat{v}}{\partial \theta} \quad \sigma_{23}=\frac{\partial \hat{v}}{\partial z}, \\
& \sigma_{31}=\frac{\partial \hat{w}}{\partial r} \quad \sigma_{32}=\frac{1}{R} \frac{\partial \hat{w}}{\partial \theta} \quad \sigma_{33}=\frac{\partial \hat{w}}{\partial z} .
\end{aligned}
$$

Introducing a new kinetic energy variable, $\hat{e}=\left(\hat{u}^{2}+\hat{v}^{2}+\hat{w}^{2}\right) / 2$, leads to (12) being re-written in the following form

$$
\begin{aligned}
\frac{\partial \hat{e}}{\partial t}+ & \frac{\partial(U \hat{e})}{\partial r}+\frac{1}{R} \frac{\partial(V \hat{e})}{\partial \theta}+\frac{1}{R} \frac{\partial(W \hat{e})}{\partial z}+\frac{U\left(\hat{u}^{2}+\hat{v}^{2}\right)}{R}+U^{\prime} \hat{u} \hat{w}+V^{\prime} \hat{v} \hat{w}+\frac{W^{\prime}\left(\hat{w}^{2}-\hat{e}\right)}{R} \\
= & -\left[\frac{\partial(\hat{u} \hat{p})}{\partial r}+\frac{1}{R} \frac{\partial(\hat{v} \hat{p})}{\partial \theta}+\frac{\partial(\hat{w} \hat{p})}{\partial z}\right]-\frac{\hat{u} \hat{p}}{R}+\frac{1}{R}\left[\frac{\partial\left(\bar{\mu} \hat{u}_{j} \sigma_{i j}\right)}{\partial x_{i}}-\bar{\mu} \sigma_{i j} \frac{\partial \hat{u}_{j}}{\partial x_{i}}\right] \\
& +\frac{1}{R}\left[\frac{\partial\left(\bar{\mu}^{\prime} \hat{u} \hat{w}\right)}{\partial r}+\frac{1}{R} \frac{\partial\left(\bar{\mu}^{\prime} \hat{v} \hat{w}\right)}{\partial \theta}+\frac{\partial\left(\bar{\mu}^{\prime} \hat{w}^{2}\right)}{\partial z}-\bar{\mu}^{\prime \prime} \hat{w}^{2}\right]+\frac{1}{R}\left[\frac{\partial\left(\bar{\mu}^{\prime} \hat{e}\right)}{\partial z}-\bar{\mu}^{\prime \prime} \hat{e}\right] \\
& +\frac{1}{R}\left[\frac{\partial\left(U^{\prime} \hat{\mu} \hat{u}\right)}{\partial z}+\frac{\partial\left(V^{\prime} \hat{\mu} \hat{v}\right)}{\partial z}+\frac{\partial\left(W^{\prime} \hat{\mu} \hat{w}\right)}{\partial z}-U^{\prime} \hat{\mu} \frac{\partial \hat{u}}{\partial z}-V^{\prime} \hat{\mu} \frac{\partial \hat{v}}{\partial z}-W^{\prime} \hat{\mu} \frac{\partial \hat{w}}{\partial z}\right] \\
& +\frac{1}{R}\left[\frac{\partial\left(U^{\prime} \hat{\mu} \hat{w}\right)}{\partial r}+\frac{1}{R} \frac{\partial\left(V^{\prime} \hat{\mu} \hat{w}\right)}{\partial \theta}+\frac{\partial\left(W^{\prime} \hat{\mu} \hat{w}\right)}{\partial z}-U^{\prime} \hat{\mu} \frac{\partial \hat{w}}{\partial r}-\frac{V^{\prime} \hat{\mu}}{R} \frac{\partial \hat{w}}{\partial \theta}-W^{\prime} \hat{\mu} \frac{\partial \hat{w}}{\partial z}-W^{\prime \prime} \hat{\mu} \hat{w}\right],
\end{aligned}
$$

which is further averaged over a single time period and azimuthal mode (removing all derivatives with respect to $t$ and $\theta$ ) and integrated across the boundary layer.

$$
\begin{aligned}
\frac{\mathrm{d} E}{\mathrm{~d} r}= & -\left\{\int_{0}^{\infty} U^{\prime}\langle\hat{u} \hat{w}\rangle+V^{\prime}\langle\hat{v} \hat{w}\rangle+\frac{W^{\prime}}{R}\left\langle\hat{w}^{2}\right\rangle \mathrm{d} z\right\}^{\mathrm{EPRS}}-\frac{1}{R}\left\{\int_{0}^{\infty} \bar{\mu}\left\langle\sigma_{i j} \frac{\partial \hat{u}_{j}}{\partial x_{i}}\right\rangle \mathrm{d} z\right\}^{\mathrm{EDV}} \\
& -\frac{1}{R}\left\{\int_{0}^{\infty}\langle\hat{u} \hat{p}\rangle \mathrm{d} z\right\}^{\mathrm{PW}}-\frac{1}{R}\left\{\int_{0}^{\infty} U\left(\left\langle\hat{u}^{2}\right\rangle+\left\langle\hat{v}^{2}\right\rangle\right)-W^{\prime}\langle\hat{e}\rangle \mathrm{d} z\right\}^{\mathrm{SC}} \\
& -\frac{1}{R}\left\{\int_{0}^{\infty} \bar{\mu}^{\prime \prime}\left\langle\hat{w}^{2}\right\rangle+W^{\prime \prime}\langle\hat{\mu} \hat{w}\rangle+U^{\prime}\left(\left\langle\hat{\mu} \sigma_{31}\right\rangle+\left\langle\hat{\mu} \sigma_{13}\right\rangle\right)+V^{\prime}\left\langle\hat{\mu} \sigma_{32}\right\rangle+W^{\prime}\left\langle\hat{\mu} \sigma_{33}\right\rangle \mathrm{d} z\right\}^{\mathrm{AVV}},
\end{aligned}
$$

where

$$
E=\int_{0}^{\infty} U\langle\hat{e}\rangle+\langle\hat{u} \hat{p}\rangle-\frac{\bar{\mu}\left(\left\langle\hat{u} \sigma_{11}\right\rangle+\left\langle\hat{v} \sigma_{12}\right\rangle+\left\langle\hat{w} \sigma_{13}+\langle\hat{u} \hat{w}\rangle\right\rangle\right)+U^{\prime}\langle\hat{\mu} \hat{w}\rangle}{R} \mathrm{~d} z .
$$

The terms labelled EPRS represent Energy Production terms due to Reynolds Stresses; EDV represent Energy Dissipation terms due to Viscosity; PW represent Pressure Work terms; SC are Streamline Curvature terms arising from the disk's rotation; and AVV are Additional terms arising from the temperature dependent (Variable) Viscosity. Note that many terms from (13) disappear upon integration due to the mean flow and perturbation boundary conditions. Here, the time-averaged quantities have the form $\langle x y\rangle=x^{\star} y+x y^{\star}$, where $x^{\star}$ indicates the complex conjugate of $x$. The perturbations retain the normal mode form, hence $r$ and $z$ derivatives can be expressed as $i \alpha \tilde{u}$ and $\tilde{u}^{\prime}$, respectively.

The energy contributions of each term in (14) are determined via numerical integration of the eigenfunctions and are shown in Figure 12 for the temperature independent, zero axial flow case $\left(\varepsilon=T_{s}=0\right)$. We see that many of the integrated terms are negligible and a simplified energy balance equation can then be expressed to give the Total Mechanical Energy (TME) as

$$
\underbrace{-2 \alpha_{i}}_{\text {TME }} \approx \underbrace{\int_{0}^{\infty} V^{\prime}\langle\hat{v} \hat{w}\rangle \mathrm{d} z}_{\text {EPRS }}-\underbrace{\frac{1}{R} \int_{0}^{\infty} \bar{\mu}\left\langle\sigma_{i j} \frac{\partial \hat{u}_{j}}{\partial x_{i}}\right\rangle \mathrm{d} z}_{\text {EDV }}
$$




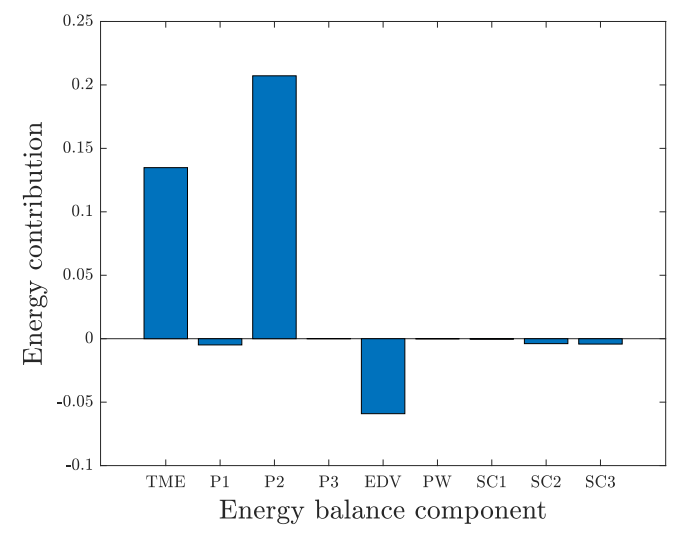

Figure 12: Results of the energy balance integral showing the energy contribution of the individual components for $\varepsilon=T_{s}=0$. Note that $\mathrm{EPRS}=\mathrm{P} 1+\mathrm{P} 2+\mathrm{P} 3$ and $\mathrm{SC}=\mathrm{SC} 1+\mathrm{SC} 2+\mathrm{SC} 3$.

where the terms have been normalised by $E$. The above returns $\alpha_{i}<0$ (the criteria for an unstable mode) when EPRS $>$ EDV. That is, a disturbance is amplified when the energy produced by the disturbance outweighs energy dissipated in response. Note that the AVV terms have not been considered here since they are equal to zero for the temperature independent (i.e. $\varepsilon=0$ ) case. The relevance of the AVV terms to (15) is discussed overleaf.

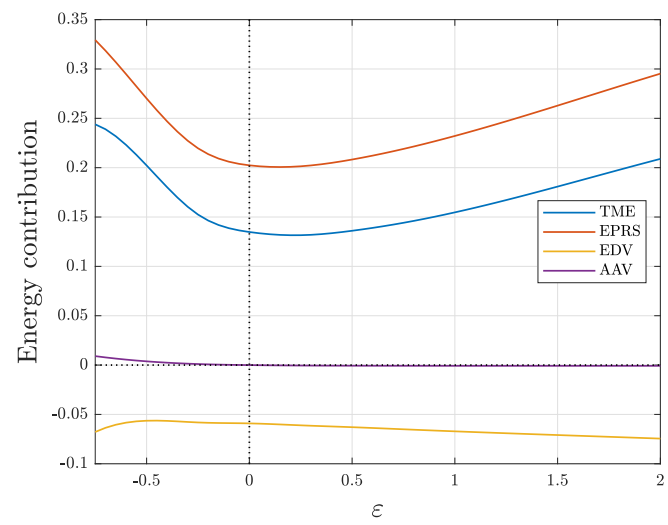

(a) Figure 13: Component energy contributions and total and component energy dissipation due to viscosity for a range
of temperature dependencies. The vertical and horizontal black, dashed lines are provided as reference to $\varepsilon=0$ and to zero energy contribution, respectively.

Figure 13(a) shows the energy integral values split into the physical components for a range of temperature dependencies. The TME curve shows that both positive and negative values of $\varepsilon$ can increase the total mechanical energy of the disturbance. Interestingly, the minimum value of TME does not occur at $\varepsilon=0$, but rather at $\varepsilon \approx 0.25$, although the TME does not change significantly in the range $0 \leq \varepsilon \leq 0.5$. The location of this minimum aligns with the $\varepsilon$ value responsible for the maximum critical Reynolds number on the $T_{s}=0$ curve in Figure 7. Further comparison between Figures 7 and 13(a) shows an inverse proportionality between $R_{c}(\varepsilon)$ and the TME. That is, a reduced critical Reynolds number also results in greater disturbance amplification. It can also be inferred that the flow stability criteria at $R_{c}$ is consistent with that at $R_{c}+200$ for all values of $\varepsilon$ considered here and as such the disturbance growth rate over this range of Reynolds numbers is unaffected by $\varepsilon$. The EDV curve shows that increasing $\varepsilon$ from $\varepsilon=-0.5$ increases the viscous dissipation associated with a disturbance. However, the gradient of the EDV curve is significantly shallower than that of the EPRS curve, leading to an overall increase of the disturbance TME. The minimum dissipation occurs when $\varepsilon=-0.5$, below this value a far greater viscous dissipation is observed. Referring back to Figure 4, we note that the $\varepsilon=-0.5$ case lacks an inflection between the type I and II branches, leading to a type II dominant instability for the cases when $\varepsilon<-0.5$. As a result, the most unstable vortex number (at which the eigenfunction is assessed) occurs on the type II branch, which may explain the departure from the trend in the otherwise type I dominant flows. We see that the additional terms that arise when $\varepsilon \neq 0$ are mostly negligible for the range of $\varepsilon$ values considered here, only adding a small amount of energy to the system for the cases when $\varepsilon<-0.5$, demonstrating that the normalised relationship in (15) is viable without the 
inclusion of the variable viscosity terms. The lack of influence of these AVV terms leads to the conclusion that the variations in the energy profiles, and hence the overall flow stability, are affected by changes to the mean flow profiles, rather than the temperature perturbations.

In order to better understand the role of temperature dependent viscosity in the flow energetics, the dissipation term is manipulated to reflect a constant viscosity component $(\bar{\mu} \equiv 1)$ and a temperature dependent component,

$$
\begin{aligned}
\underbrace{-\frac{1}{R} \int_{0}^{\infty} \bar{\mu}\left\langle\sigma_{i j} \frac{\partial \hat{u}_{j}}{\partial x_{i}}\right\rangle \mathrm{d} z}_{\text {EDV }}= & -\underbrace{\frac{1}{R} \int_{0}^{\infty}\left\langle\sigma_{i j} \frac{\partial \hat{u}_{j}}{\partial x_{i}}\right\rangle \mathrm{d} z}_{\mathrm{ND}} \\
& -\underbrace{\frac{1}{R} \int_{0}^{\infty}(\bar{\mu}-1)\left\langle\sigma_{i j} \frac{\partial \hat{u}_{j}}{\partial x_{i}}\right\rangle \mathrm{d} z}_{\text {TDD }}
\end{aligned}
$$

Here, the term labelled ND represents Newtonian Dissipation and the term labelled TDD represents Temperature Dependent Dissipation. The terms established in (16) are plotted in Figure 13(b). As $\varepsilon$ is increased, the Newtonian dissipation briefly reduces, before increasing significantly. Conversely, the dissipation associated with the temperature dependent component is seen to decrease quasi-linearly as $\varepsilon$ increases, where TDD becomes positive when $\varepsilon>0$, acting as an energy production term and reducing the overall viscous dissipation.

Figure 14 shows the energy integral values for a range of axial flow strengths. Unlike the temperature dependent viscosity, the formulation of enforced axial flow used here does not change the stability equations from the standard von Kármán formulation. Hence, it is known that any variation in the stability of the flow is a result of variations of the mean-flow profiles. We see that as $T_{s}$ is increased, each of the curves moves towards zero; the energy produced by the disturbance is decreased and the viscous dissipation decreases in turn, reducing the total mechanical energy. The results seen here are consistent with the stabilising effect of increasing $T_{s}$ demonstrated in Figure 5, where higher Reynolds numbers are required to amplify a disturbance sufficiently to cause instability.

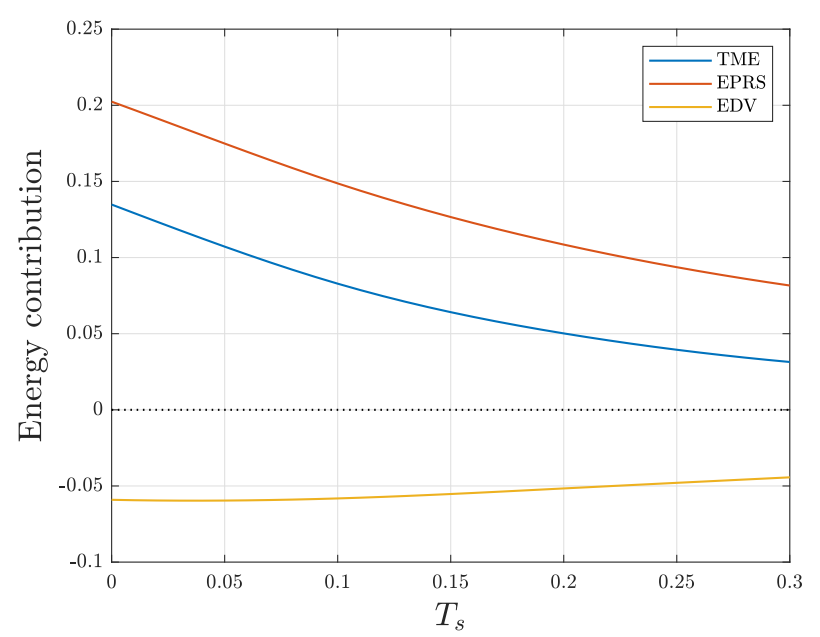

Figure 14: Component energy contributions for a range of axial flow strengths.

Figure 15 shows the energy contribution of each component of the energy balance equation for a range of temperature dependencies at three fixed axial flow strengths. We see that the general trend resulting from increasing $\varepsilon$ remains unchanged when enforced axial flow is applied. In the case when $T_{s}=0.15$, each curve is notably shallower, which is consistent with the dampening effect of enforced axial flow seen in Figure 14. The $T_{s}=0.3$ curves are shallower still, where overall variation appears to be largely insignificant. However, recalling Figure 6 we observe that the critical Reynolds number does in fact vary significantly for a range of $\varepsilon$ values when $T_{s} \neq 0$. As such, enforced axial flow cannot fully suppress the destabilisation associated with temperature dependent viscosity flows, it is, however, helpful in dampening the growth of the disturbances.

\section{Discussion and conclusions}

In $\S 2$, the formulation and solution of the mean-flow equations were presented for a range of temperature dependent viscosities and enforced axial flow strengths. We found that negative values of the temperature 

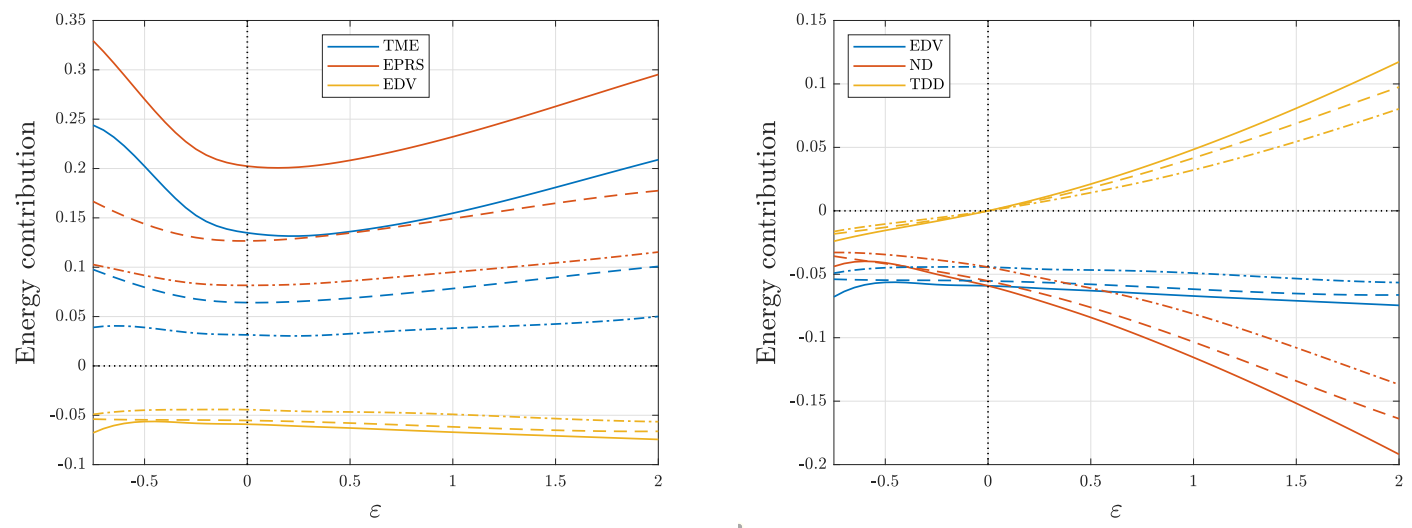

Figure 15: Component energy contributions \& total and component energy dissipation due to viscosity for a range of temperature dependencies. Solid lines $(-)$ represent $T_{s}=0$; dashed lines $(--)$ represent $T_{s}=0.15$; dash-dot lines (-.) represent $T_{s}=0.3$.

dependence value $\varepsilon$, result in a steady convergence of the velocity and temperature profiles to their respective boundary conditions as $z \rightarrow \infty$, indicating a broader boundary-layer thickness. Conversely, positive values of $\varepsilon$ result in convergence of the profiles at a reduced distance from the disk surface and therefore a narrower boundary layer. The physical relation between the temperature dependency and the boundary layer thickness can be described through the viscosity at the wall and its relation to the wall shear, where changes to the viscous forces here affect the impact of the disk's rotation on the surrounding fluid.

Increasing the axial flow strength results in a reduced convergence distance of the mean flow profiles with the exception of the axial velocity profile, which - as to be expected for forced flow in this direction - exhibits acceleration throughout the boundary layer. An enforced flow results in a gentle narrowing of the boundary layer as the influence of the disk's rotation is diminished.

When combining the two parameters, the effects they exhibit individually upon the mean flow are generally consistent when applied simultaneously. Notable exceptions are the effects upon the axial velocity and temperature profiles $(W(z)$ and $\Theta(z)$, respectively). Beyond a certain axial flow strength, the effect of increasing the temperature dependence is reversed. More specifically, when $T_{s}=0.3$ the axial velocity is increased with increasing $\varepsilon$, and the temperature profile converges at a reduced distance (i.e. the thermal boundary layer is narrowed). This is due to the non-zero convergence of the radial velocity profile when $T_{s} \neq 0$, which then inhibits the characteristic profile inflection.

Having detailed the physics behind the mean-flow behaviour and its variation with temperature dependence and enforced axial flow, it is pertinent to apply the same to the observed effects with regards to flow stability. In $\S 3$ we observed that solutions to the stability equations can be plotted in the form of neutral stability curves, where the stability of the flows can be assessed primarily through the location of the critical Reynolds number. It was found that negative values of $\varepsilon$ lead to significantly destabilised flows when compared to the temperature independent case. As $\varepsilon$ is increased, the type I critical Reynolds number increases and the resulting flows are more stable. When comparing this behaviour to that of the mean flow, one might conclude that the narrowing of the boundary layer is favourable to flow stability. However, increasing $\varepsilon$ into the positive regime continues this stabilising trend, up until a point where beyond which the critical Reynolds number reduces, eventually to a value less than that of the temperature independent case $(\varepsilon \approx 0.6$ as detailed in Figure 7). The type II mode appears to be destabilised for all non-zero values of $\varepsilon$ considered, suggesting that any variation in the viscosity through the boundary layer will promote this instability.

The stability response to variations in axial flow strength have also investigated. We find that the type I mode is stabilised significantly as the $T_{s}$ is increased. Again, referring to the mean flow suggests that the narrowing of the boundary layer caused by increasing $T_{s}$ is favourable to the stability of the type I mode. Referring back to Figure 2 shows that the characteristic inflection of the radial profile is inhibited as $T_{s}$ is increased due to the boundary condition on $U(z)$. This inflection is physically responsible for the crossflow instability; hence, if the converging velocity beyond the inflection is closer to the profile maximum, the instabilities arising from the velocity gradient should be less significant. It is also noted in Figure 2 that, when $T_{s}=0.3$, the inflection is completely suppressed. However, Hussain et al. [16] note that an inflection exists in the resolved velocity profile ${ }^{\dagger}$ for all values of $T_{s}$, and hence a crossflow instability will always arise.

The type II behaviour is more difficult to characterise physically. The eventual stabilising effect of

${ }^{\dagger}$ The resolved velocity $Q=U \cos \phi+V \sin \phi$, where $\phi$ is the resolution angle from the radial profile in the direction of rotation. 
increasing $T_{s}$ can be explained as the forced flow suppressing the influence of the disk's rotation (from which the type II instability arises). However, the small range of $T_{s}$ values where increasing $T_{s}$ is destabilising to the type II mode falls outside of this explanation. Recall that the type II mode is viscous in nature; perhaps this weakly enforced axial flow encourages the viscous interaction between the fluid and the disk.

We have also assessed the radial growth rate of disturbances for a range of temperature dependencies and axial flow strengths, utilising them to find disturbance amplitude ratios and plotting $N$-factor curves that approximate the location of a transitional Reynolds number. We find that the lowest transitional Reynolds numbers correspond to the $\varepsilon$ values that produce the least stable flows, i.e. $\varepsilon=-0.75$. Increasing the enforced axial flow here significantly increases the transitional Reynolds number, as well as suppressing the growth rate of the $N$-factor curve. The overall behaviour largely imitates that of the neutral curves. As such, the physical mechanisms that allow instabilities to form at lower Reynolds numbers are likely also responsible for the development of these instabilities into fully turbulent flow over a reduced distance. A notable result is that enforced axial flow may still delay transition in spite of the destabilising effect of increasing $\varepsilon$ beyond its most stable value; the reduced gradient of the $N$-factor curves induced by enforced axial flow means that the development of disturbances from instability to turbulence is delayed, and therefore a reduced critical Reynolds number is not necessarily indicative of an earlier onset of transition. Experimental work is required to verify this, but the results presented here imply that, for certain cases, the radial position where crossflow vortices form could occur closer to the disk centre, whilst the turbulent region develops further downstream.

In §3.5, the magnitudes of the perturbation eigenfunctions were plotted and an energy balance equation was derived from the stability equations. The total energy of the system follows the trend established between the critical Reynolds number and $\varepsilon$, where the minimum disturbance energy and the maximum critical Reynolds number approximately coincide when $\varepsilon \approx 0.25$. A particularly interesting result is that the additional disturbance terms arising from the inclusion of a temperature dependent viscosity are found to be negligible. We therefore conclude that the linear stability characteristics of such flows is predominantly governed by variations in the basic states. It is established in (15) that the energy production term (EPRS) is dependent on $V^{\prime}(z)$ and the viscous dissipation term (EDV) is dependent on $\bar{\mu}(z ; \Theta(z))$. With this in mind, recall the mean flow solutions from $\S 2$. As $\varepsilon$ is increased, the gradient of the azimuthal profile in Figure 1 increases as the profile is narrowed, leading to a significant change in the shape of the $V^{\prime}(z)$ profile. This change in the $V^{\prime}(z)$ profile is clearly favourable as $\varepsilon$ is increased in the range $-0.75 \leq \varepsilon \leq 0.25$ (where the upper limit is the point of maximum stability), reducing the energy production term and thereby creating a more stable flow. Perhaps beyond this, the steep velocity gradients through a narrow boundary layer render the flow more susceptible to instability.

It has already been suggested that certain mean flow behaviours - such as the suppression of the radial profile inflection - are in part responsible for the stabilising effect. It is seen in Figure 14 that increasing $T_{s}$ leads to each profile moving towards zero. Examining the $V^{\prime}(z)$ profile behaviour with increasing $T_{s}$ in Figure 2 shows that although the absolute value of $V^{\prime}(0)$ is increasing (see also Table 1), the profile is also narrowed such that the area enclosed by the $V^{\prime}(z)$ curve is reduced. As such, the energy produced by the disturbance is significantly reduced. Figure 15 shows that this effect is consistent for all temperature dependencies considered here. Enforced axial flow evidently acts as a dampener to the amplification of disturbances.

Where values match, the neutral curve data presented here for varying enforced axial flow is in excellent agreement with the data presented in Hussain et al. [16]. An alternative forced flow formulation is presented in Chen \& Mortazavi [3], where the mean flow of a chemical vapour deposition reactor is modelled. The forced flow parameters are expressed as modifications to the mean flow variables $U(z)$ and $W(z)$, where two constants control the ratio of rotational to axial influence on the flow. The formulation utilised by Chen \& Mortazavi [3] allows for all flows between the standard, rotation-only von Kármán flow and forced flow-only stagnation flow. The effects observed on the mean flow are consistent with those seen here, where the radial inflection is suppressed due to the imposed boundary condition. As noted by Hussain et al. [16], the advantage of the formulation utilised in this work is that $T_{s}$ does not appear in the stability equations, whilst the formulation used by Chen \& Mortazavi [3] would modify the stability equations. However, the formulation utilised here is only suitable for enforced axial flow strengths where rotation is dominant. As such, to extend this analysis to account for weak rotational influence on the forced flow over a disk (i.e. $T_{s}>1$ ), a formulation similar to that of Chen \& Mortazavi [3] would be more suitable.

We have limited the work presented here to stationary modes of instability that rotate with the disk surface. The most obvious extension of this work would be to investigate travelling modes. Typically, travelling modes yield higher critical Reynolds numbers with reduced amplification rates; hence stationary modes are often the primary source of linear convective instability. This was first detailed by the visualisation experiments of Gregory et al. [11], where the existence of travelling modes was verified theoretically, but stationary vortices were observed on china clay disks. However, experimental work [6] has since shown highlighted the importance of travelling modes over a smooth and clean disk surface. 
The behaviour of travelling modes in the formulation presented here can be speculated from the results of previous studies. For temperature dependent viscosity flows (without axial forcing), Jasmine \& Gajjar [19] find that for all values of $\varepsilon$ considered, increasing the frequency of the disturbance is mildly stabilising to the type I modes, whilst highly destabilising with respect to the type II mode. Similarly, for flows with axial forcing (without a temperature dependent viscosity), Hussain et al. [16] find that increasing the disturbance frequency is mildly stabilising to the type I mode and heavily destabilising to the type II mode. They also investigate some negative frequencies, which are indicative of a disturbance travelling slower than the disk: these modes are found to be the most amplified, though it is suggested that less-amplified near-stationary frequencies are more likely to be selected due to a lower critical Reynolds number. It may therefore be expected that travelling modes in this formulation would most likely appear when $\varepsilon \ll 0$ and $T_{s} \approx 0$, as the stationary modes at these parameter values are the most amplified according to the amplitude ratio curves and integral energy analysis (Figures 9 and 15, respectively).

RM would like to thank the EPSRC for funding this research (award reference 1658206).

\section{References}

[1] AlveroĞLu, Burhan 2016 The convective instability of the BEK system of rotating boundary-layer flows over rough disks. PhD thesis, University of Leicester.

[2] Bertolotti, F. P., Herbert, Th. \& Spalart, P. R. 1992 Linear and nonlinear stability of the Blasius boundary layer. Journal of Fluid Mechanics 242, 441-474.

[3] Chen, K. \& Mortazavi, A. R. 1986 An analytical study of the chemical vapor deposition (CVD) processes in a rotating pedestal reactor. Journal of Crystal Growth 76 (1), 199-208.

[4] Chomaz, J. 2005 Global instabilities in spatially developing flows: non-normality and nonlinearity. Annu. Rev. Fluid Mech. 37, 357-392.

[5] Cooper, A. J. \& CArpenter, P. W. 1997 The stability of rotating-disc boundary-layer flow over a compliant wall. part 1. type I and II instabilities. Journal of Fluid Mechanics 350, 231-259.

[6] Corke, T. C. \& Knasiak, K. F. 1998 Stationary travelling cross-flow mode interactions on a rotating disk. Journal of Fluid Mechanics 355, 285-315.

[7] Cossu, C., Chomaz, J. \& Henningson, D. S. 2001 2D nonlinear front propagation in the Blasius boundary layer. In APS Division of Fluid Dynamics Meeting Abstracts, , vol. 54.

[8] Fedorov, B. I., Plavnik, G. Z., Prokhorov, I. V. \& Zhukhovitskit, L. G. 1976 Transitional flow conditions on a rotating disk. Journal of Engineering Physics and Thermophysics 31 (6), 1448-1453.

[9] Garrett, S. J., Hussain, Z. \& Stephen, S. O. 2010 Boundary-layer transition on broad cones rotating in an imposed axial flow. AIAA Journal 48 (6), 1184-1194.

[10] Gray, W. E. 1952 The nature of the boundary layer flow at the nose of a swept wing. RAE TM Aero 256.

[11] Gregory, N., Stuart, J. T. \& Walker, W. S. 1955 On the stability of three-dimensional boundary layers with application to the flow due to a rotating disk. Phil. Trans. R. Soc. Lond. A 248 (943), 155-199.

[12] Hall, P. 1986 An asymptotic investigation of the stationary modes of instability of the boundary layer on a rotating disc. Proceedings of the Royal Society of London. A. Mathematical and Physical Sciences 406 (1830), 93-106.

[13] Healey, J. J. 2004 On the relation between the viscous and inviscid absolute instabilities of the rotating-disk boundary layer. Journal of Fluid Mechanics 511, 179-199.

[14] Holstein, W. L. 1992 Design and modeling of chemical vapor deposition reactors. Progress in Crystal Growth and Characterization of Materials 24 (2), 111-211.

[15] Huerre, P. \& Monkewitz, P. A. 1990 Local and global instabilities in spatially developing flows. Annual Review of Fluid Mechanics 22 (1), 473-537. 
[16] Hussain, Z., Garrett, S. J. \& Stephen, S. O. 2011 The instability of the boundary layer over a disk rotating in an enforced axial flow. Physics of Fluids 23 (11), 114108.

[17] VAN IngEn, J. L. 1956 A suggested semi-empirical method for the calculation of the boundary layer transition region. Tech. Rep.. Delft University of Technology.

[18] VAN InGEN, J. L. 2008 The $e^{N}$ method for transition prediction. historical review of work at tu delft. In 38th Fluid Dynamics Conference and Exhibit, p. 3830.

[19] Jasmine, H. A. \& GajJAR, J. S. B. 2005 Absolute and convective instabilities in the incompressible boundary layer on a rotating disk with temperature-dependent viscosity. International Journal of Heat and Mass Transfer 48 (5), 1022-1037.

[20] Jensen, K. F., Einset, E. O. \& Fotiadis, D. I. 1991 Flow phenomena in chemical vapor deposition of thin films. Annual Review of Fluid Mechanics 23 (1), 197-232.

[21] Kafoussias, N. G. \& Williams, E. W. 1995 The effect of temperature-dependent viscosity on freeforced convective laminar boundary layer flow past a vertical isothermal flat plate. Acta Mechanica 110 (1), 123-137.

[22] VON KÁRMÁn, T. 1921 'Uber laminare und turbulente reibung. ZAMM - Journal of Applied Mathematics and Mechanics / Zeitschrift für Angewandte Mathematik und Mechanik 1 (4), 233-252.

[23] Kleijn, C. R. 1991 On the modelling of transport phenomena in chemical vapour deposition and its use in reactor design and process optimization. Thin Solid Films 206 (1-2), 47-53.

[24] Ling, J. X. \& DyBbs, A. 1992 The effect of variable viscosity on forced convection over a flat plate submersed in a porous medium. Journal of heat transfer 114 (4), 1063-1065.

[25] Lingwood, R. J. 1995 Stability and transition of the boundary layer on a rotating disk. PhD thesis, University of Cambridge.

[26] Lingwood, R. J. 1996 An experimental study of absolute instability of the rotating-disk boundary-layer flow. Journal of Fluid Mechanics 314, 373-405.

[27] Lingwood, R. J. \& Alfredsson, P. H. 2015 Instabilities of the von Kármán boundary layer. Applied Mechanics Reviews 67 (3), 030803.

[28] Lloyd, J. R. \& Sparrow, E. M. 1970 On the instability of natural convection flow on inclined plates. Journal of Fluid Mechanics 42 (3), 465-470.

[29] Malik, M. R. 1986 The neutral curve for stationary disturbances in rotating-disk flow. Journal of Fluid Mechanics 164, 275-287.

[30] Malik, M. R., Wilkinson, S. P. \& Orszag, S. A. 1981 Instability and transition in rotating disk flow. AIAA Journal 19 (9), 1131-1138.

[31] Miller, R., Garrett, S. J., Griffiths, P. T. \& Hussain, Z. 2018 Stability of the Blasius boundary layer over a heated plate in a temperature-dependent viscosity flow. Phys. Rev. Fluids 3, 113902.

[32] Schlichting, H. 1933 Laminare strahlausbreitung. ZAMM - Journal of Applied Mathematics and Mechanics / Zeitschrift für Angewandte Mathematik und Mechanik 13 (4), 260-263.

[33] Tollmien, W. 1929 Über die entstehung der turbulenz. Nachr. Ges. Wiss. G“öttingen Math.-Phys. Kl. II, 21-44.

[34] Wall, D. P. \& Wilson, S. K. 1997 The linear stability of flat-plate boundary-layer flow of fluid with temperature-dependent viscosity. Physics of Fluids 9 (10), 2885-2898.

[35] Wilkinson, S. P. \& Malik, M. R. 1985 Stability experiments in the flow over a rotating disk. AIAA journal 23 (4), 588-595. 